\title{
The 3-D Positioning of Wireless Sensors in Dispersive Propagation Media
}

\author{
J.G Davis, R. Sloan, Senior Member IEEE and A.J Peyton
}

\begin{abstract}
This paper describes a novel inversion algorithm for determining the 3-D position coordinates and environmental complex permittivity of wireless nodes embedded in a dispersive propagation media. In this study, wireless sensors are considered to form part of a network for monitoring salient parameters such as temperature and humidity in large wheat filled storage vessels, where the highly reflective nature of the propagation environment engenders extreme multipath effects. Under such conditions, the inherent difficulties in deriving reliable pulse propagation delay and amplitude estimates, greatly diminishes the effectiveness of conventional free-space positioning methods. These limitations have lead to the development of alternative inversion techniques capable of retrieving both geometric positioning and electromagnetic propagation data. The inversion algorithm developed within, is formulated as a nonlinear least squares problem in which the misfit between time domain positioning measurements and modelled data is minimized via application of a novel minimum single level linkage optimisation algorithm. In developing a more refined propagation forward solution incorporating position coordinates, media complex permittivity and antenna radiation pattern acceptable positioning resolutions and macroscopic material property estimates have been obtained under challenging propagation conditions,
\end{abstract}

Index Terms - positioning, ultra wideband, wireless sensor.

\section{Introduction}

The deployment of wireless sensor networks within industrial and military applications for remote scientific monitoring purposes is becoming increasingly common place. Sensor networks have the capability to generate salient parameters such as temperature, pressure and humidity within physically in-accessible regions or extremely hazardous industrial environments such as nuclear or chemical installations. Wireless sensor network applications have been extended to include the subterranean tomographic imaging of 
geological features and the mapping of physical parameters within grain silos or chemical storage vessels. A prerequisite of any sensor network is the capability to provide the in-situ 3-D positioning of at least three individual sensors within the operating environment such that a network based positioning framework may be maintained. In certain classes of networking operation conditions, wireless sensors will be submerged in inhomogeneous substances such as soil, as in Ground penetrating radar, cross-borehole applications [1], or wheat in grain-silo temperature and humidity mapping investigations [2]. Sensor node positioning here presents a greater challenge, due to a lack of knowledge of the propagation media phase velocity and attenuation and the presence of submerged scattering obstacles such as vessel walls or pipes. The ultra-wideband 3-D positioning technique described here adopts classic tomographic methods to locate wireless sensor nodes immersed in wheat under such rich multi-path conditions. The study adapts the previous equivalent free-space space methodology [3, 4], where sensor node positioning was conducted over an empty cylindrical vessel, by scanning a single antenna along a set of orthogonal synthetic apertures above a wheat filled vessel. As per their free-space counterpart methods, advances in UWB pulse positioning techniques [5], are exploited to facilitate single or 'stand alone' sensor localization in the presence of multi-path, via implementation of a novel line-of-sight isolation technique. However, as a further refinement, in this wheat based application, the pulse spectral power density is customised to reduce the level of signal dispersion within the lossy wheat media and provide sufficient resolution to isolate the desired line-of-sight propagation component. Furthermore, Gaussian post-processing filtering is applied to minimise sidelobe induced perturbation effects a feature of particular necessity for positioning estimates in lossy propagation media.

Ultimately, the resolving power and range of the positioning architecture are dictated by the frequency content of the pulse and the loss tangent of the propagation medium [6]. Consequently, although wide bandwidth electromagnetic pulses exhibit greater resolution in free space, this parameter along with the pulse penetration depth, may be reduced considerably due to differential attenuation of the higher frequency spectral components. Hence, a trade-off between these factors requires a considered choice of the UWB sensor communication architecture, in conjunction with pre-emphasis of the pulse power spectrum higher frequency region. In essence, full consideration of the prevailing physical mechanisms is essential if pulse propagation characteristics are to be correctly factored into the positioning algorithm and the sensor location determined. Hence, errors in propagation 
delay, pulse dispersion and sensor antenna directivity arising from the unknown nature of the propagation environment must be addressed as per Ground penetrating radar (GPR) applications [7]. The ultra-wideband 3-D positioning technique described here applies tomographic inversion methods to locate sensor nodes under multipath conditions whilst simultaneously computing the complex propagation constant and sensor antenna directivity. This has been accomplished via the formulation of an adapted Friis propagation model incorporating the relative geometric location of the wireless emitter, the complex permittivity of the propagation medium and radiation pattern of the embedded antennas.

The novelty of this approach is twofold. Firstly, an ultra-wideband pulse edge detection method is combined with spatially diverse synthetic aperture measurements to isolate the line-of-sight component from unwanted vessel wall induced multipath interference. Secondly, an iterative inversion process determines the optimum transfer function for each measurement position. This process minimises the difference between the measured and reconstructed pulse waveforms and replicates the original measured counterparts [8, 9]. The location of the inversion global minima within the optimised $P$-dimensional parameter space yields the true position coordinates and electrical properties of the host medium - a process analogous to focussing in imaging applications. These additional optimisation features combine to facilitate both precision positioning and cumulative error source estimation, yielding down and cross range resolutions approaching $6 \mathrm{~cm}$ in rich scattering environments. The results of the application of this new inversion technique compare favourably with the physically measured sensor coordinates and published data for the RF propagation characteristics of wheat. In summary, the contents of the paper may be subdivided into four sections comprising: a description of the synthetic aperture positioning methodology, an overview of the modified propagation forward solution within dispersive media, a description of the inversion algorithm used to derive the sensor positioning and complex permittivity data, and finally, a comparison between results derived from the inversion process with those obtained via measurement.

\section{The Positioning Measurement Architecture}

The experimental configuration illustrated in Fig. 1 shows the network architecture of the 3-D tomographic inversion and ultra wideband positioning system for a wheat filled grain silo. Here a $5 \mathrm{~V}$ step generator rising edge is converted into a $50 \mathrm{mV}$ peak Gaussian pulse 


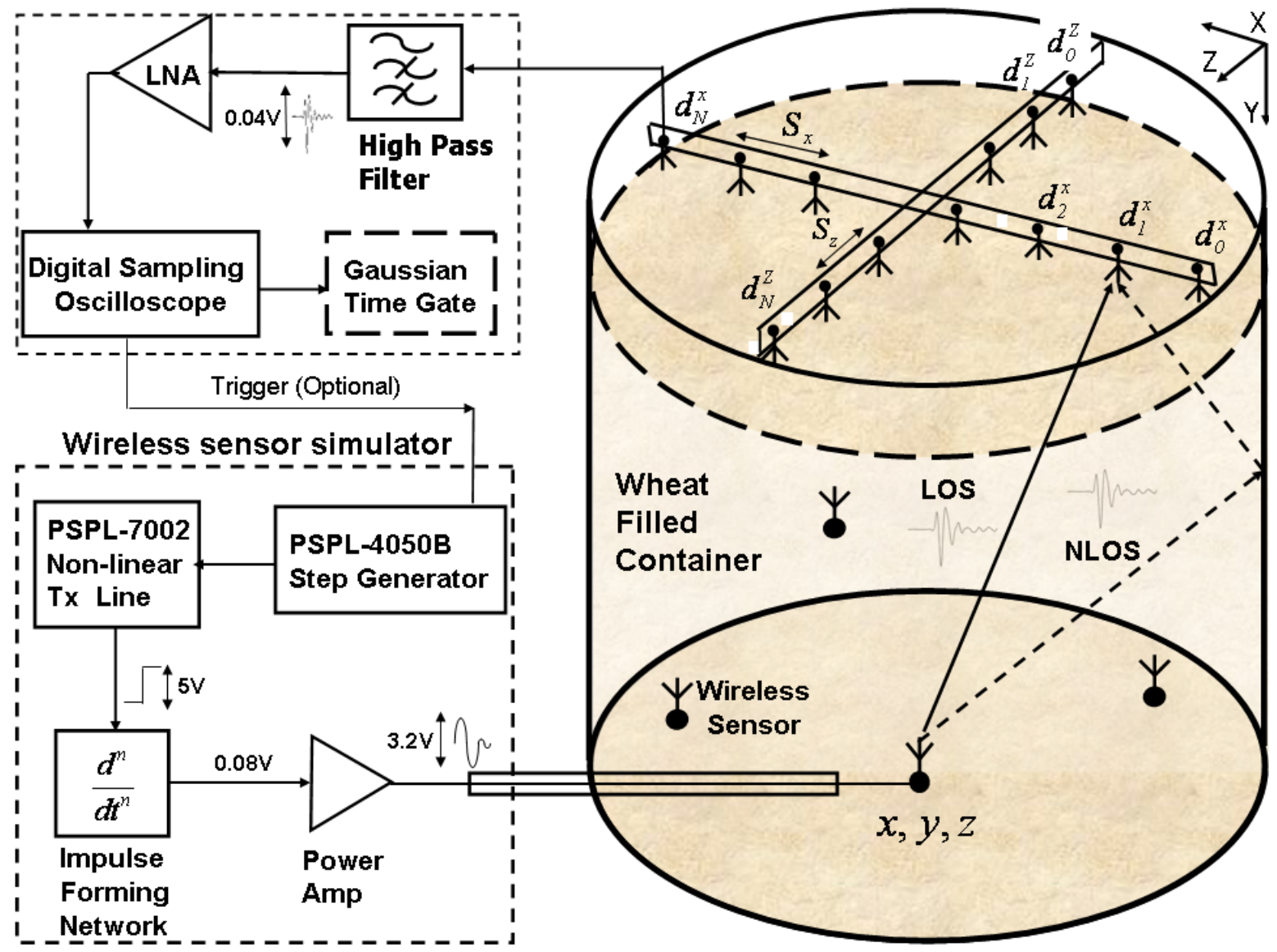

Fig. 1: 3-D Ultra wideband positioning in a wheat based rich scattering envir onment using a wireless sensor node forward transmission path simulator incorporating Gaussian weighted time gating.

$1^{\text {st }}$ derivative or 'monocycle' via a combination of pulse generator, non-linear transmission line and cascaded impulse forming networks. Further amplification by a $30 \mathrm{~dB}$ gain LNA, augments the signal to a $3.5 \mathrm{~V}$ peak to peak amplitude pulse prior to transmission via ultrawideband ring monopole transmitting antenna [10]. The pulse generation system has been optimised via prior experimentation with successive cascaded impulse forming networks to yield the order of Gaussian derivative containing spectral density profiles where $95 \%$ of the power is concentrated within a 0.5 to $2.5 \mathrm{GHz}$ bandwidth - a frequency range considered optimal for low-loss propagation in wheat. Note, the effect of increasing the derivative order here, systematically displaces the power spectra profile up in frequency, a useful technique for customising the Gaussian derivative pulse shapes used to conform with FCC regulations [25]. The bandwidth of the UWB annular ring monopole antenna, discussed in greater detail in Section 3, has also been designed to accommodate its monocycle excitation pulse thereby maximising propagation efficiency. An identical reciprocal antenna, positioned at discrete intervals along the two orthogonal supports displayed in Fig 
3 , forms the synthetic aperture receive array. This antenna, in measuring the $1^{\text {st }}$ derivative of the transmitted pulse at each array measurement position, yields a composite signal containing both line-of-sight and non-line-of-sight components attributable to reflections from the vessel floor and walls etc. Calculation of the transmitter position is effected via the determination of the emitter to array sensor transfer functions required to reconstitute the series of time pulses recorded at each array sensor position. The key to the success of this location technique is the isolation of the pulse waveform purely attributable to direct line of sight (LOS) transmission. This LOS region is identified via measurement at up to 10 spatially diverse locations and isolated via application of a temporal band pass filter or 'range gate' (Fig. 2).

The prototype architecture of Fig 1 simulates the transmission action of a typical transceiver in a wireless sensor network, which in future may encompass independently powered sensor nodes, containing ultra wideband monocycle pulse generator, power amplifiers and conformal UWB antenna. At the receiver, heavily attenuated $\sim 30 \mathrm{mV}$ peak pulse time domain waveforms are recorded, after additional amplification from a ZX602522M LNA, by an Agilent 84558A, 20 GSa/s digitising oscilloscope, with a memory depth of $1.023 \times 10^{6}$ samples per channel. In positioning experiments with the sampling oscilloscope, the acquisition and synchronisation of a stable, low jitter, triggering reference signal, was achieved for a single sensor either directly using a 'hard wired' cable connection or remotely via a second scanning array antenna connected to the oscilloscope auxiliary input via high pass filter and low noise amplifier. Note, for future multiple sensor measurements, time division coded sequences may be sent to each sensor sequentially via a UHF transmitter link to activate it whilst triggering the sampling oscilloscope [11]. The ability to remotely acquire and trigger via a simulated sensor pulse train confirmed that true asynchronous positioning is attainable in this noisier, high absorption wheat based propagation scenario, a notable advance on the free-space positioning system of [4,5].

The positioning measurements were conducted by moving the single linearly polarized ring monopole receive antenna sequentially at $5 \mathrm{~cm}$ intervals over $2.0 \mathrm{~m}$ long, orthogonal apertures positioned approximately $2 \mathrm{~cm}$ above the surface of wheat. Four transmit positions were tested in total, by submerging the ring monopole antennas, (attached to fibre glass poles for ease of manoeuvering) at different locations within the wheat filled container. The vertical displacement of the sensors, as measured from the origin of the cartesian coordinate system in Fig. 1, were fixed at $(1.00,0.95,1.05 \& 0.80 \mathrm{~m})$ 
respectively. The vessel itself, comprising a plastic tank of diameter $2.0 \mathrm{~m}$ and height 1.8 $\mathrm{m}$, possessed walls of sufficient reflectivity $(\rho \sim 0.25)$ to ensure the presence of a significant multi-path reflections within the volumetric wheat space. The overall effective angular displacement of the synthetic positioning aperture across its width $\left(\sim 30^{\circ}\right)$, ensured a worst case variation in cross range resolution of $6 \mathrm{~cm}$, with a down range resolution, as dictated by the bandwidth of the transmitted monocycle pulse and target detection range, varying between approximately $3 \mathrm{~cm}$ and $6 \mathrm{~cm}$ over the vessel depth.

\section{A. Application of temporal range gate filtering}

Examples of typical time domain responses arising from monocycle pulse excitation are shown in Fig. 2 for a series of measurements made along the $\mathrm{X}$-axis. The received waveform structure comprises a series of impulse responses attributable to direct LOS excitation of the receiving sensor and other multi-path components, subjected to an overall dispersive pulse broadening effect arising from the lossy characteristics of the wheat based propagation medium. In order to isolate the effective portion of the time domain response attributable to LOS propagation, a temporal Gaussian shaped filter $G(t)$ was applied to the measured waveform $S_{n}(t)$ at each array position to 'range gate' the leading edge portion of the waveform. The Gaussian post processing temporal filter, was chosen to minimise the generation of any side lobes in the equivalent frequency domain waveform, arising from 'time-gating' truncation effects. This feature - an enhancement of the original rectangular pulse post-processing architecture in [1] - is of particular importance in lossy media positioning measurements, as excessive sidelobe distortion may affect the fidelity of the system impulse response reconstruction and with it the accuracy of the sensor position estimates. The resulting sequence of gated pulses $V(t)$ is hence given by:

$$
V(t)=G(t-\Delta t) \cdot\left\{S_{1}(t), S_{2}(t), \ldots \ldots \ldots, S_{n}(t)\right\} \quad ; \quad G(t)=e^{-\left(\frac{t}{\sqrt{2} \sigma}\right)^{2}}
$$

where $\Delta t$ is the propagation delay of the $n^{\text {th }}$ pulse and $\sigma^{2}$ the Gaussian pulse variance. The sampling process is illustrated in Fig. 2, where the dashed rectangular box represents the portion of the waveform to be isolated with the Gaussian range gate. When applying the sampling algorithm, gated sections of the pulses monitored at all $N$ receiver positions along 


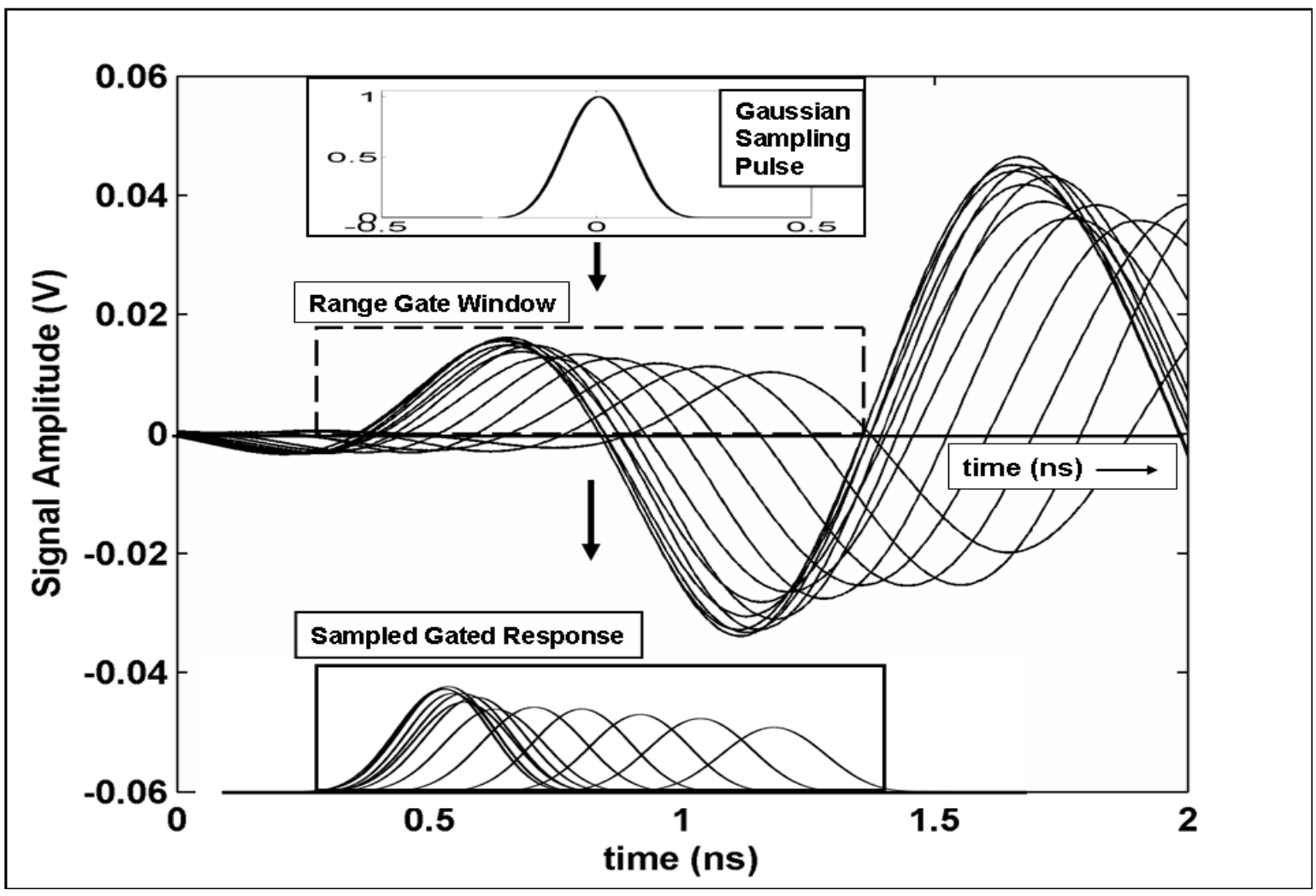

Fig. 2 : Gaussian Range gate sampling of received X-axis synthetic aperture measurements after pulse excitation of the wheat embedded ring monopole transmitting sensor.

the orthogonal $X$ and $Z$-axes, are recorded using the $20 \mathrm{GSa} / \mathrm{sec}$ digitising oscilloscope and averaged over a total of 16 snapshots. An example of the ensuing series of delayed Gaussian pulses along with the original waveforms from which they were derived are provided in Fig. 5(a) \& (b).

\section{B. Gaussian pulse post processing}

The rationale for filtering the original portion of the waveform, to isolate the pure LOS propagation region, arose from a consideration of the overall system impulse response. For an infinite bandwidth and lossless medium, any measurement would include a unique series of impulses, comprising an initial LOS component followed by subsequent multipath components. Further evolution of this model via reduction in bandwidth and an increase in attenuation, will transform these into the indistinguishable, set of overlapping waveforms illustrated in Figure 2. However, a strategically positioned 1.0 ns width range gate filter, should, on consideration of the propagation path geometry of Fig. 1, encompass the temporal region attributable to LOS propagation only. As the same set-up geometry applies 
to the measured and reconstructed data, the position coordinates and host permittivity may be retrieved, providing identical filtering and transformation algorithms are applied to both. It is the RMS difference between reconstructed and measured data, obtained via a direct comparison between their respective pulse sequences, which forms the basis of the inversion algorithm error function detailed in Section 4.

On analysing the series of pulses generated via application of the range gate window in Fig. 2, the propagation characteristics, (not immediately evident in the more cluttered, nongated original waveforms) now become clear. Here, the three fundamental dispersion related phenomena, intrinsically linked to the emitter position and wheat permittivity are evident; namely: the displacement in time, the relative change in pulse amplitude and overall broadening of the pulse shape. The purpose of the inversion exercise then is to determine the emitter position coordinates, host medium permittivity and associated set of transfer functions which minimise the difference between measured and reconstructed pulse waveforms and replicate the aforementioned pulse propagation features.

\section{Ultra wideband Propagation and Positioning in Wheat}

The ultimate objective of this analysis is the determination of the sensor position coordinates in dispersive wheat based propagation media via implementation of an inversion algorithm. This is realisable via inversion methods, providing a forward solution be derived capable of emulating the propagation delay, attenuation and dispersion conditions required for reproduction of the complete set of Gaussian filtered pulses from the measured response at any given array position. Note, this constitutes a significant enhancement over the air-spaced positioning methodology in [3], which considered propagation delay only. As prior knowledge of the host medium permittivity and loss tangent are required to achieve this goal, the resulting algorithm, out of necessity included the propagation loss within the wheat based propagation medium as an additional optimisation variable, thus enabling the medium complex permittivity to be derived as a by-product. The first step in realising this objective was the development of a series of transfer functions describing the dispersive nature of the propagation paths between emitter and array sensor, as is now detailed. 


\section{A. Modified Friis propagation equation in wheat}

Application of the Fraunhofer near to far field boundary criteria to the co-polar aligned sensor antennas in Fig. 3, with a 0.15 m largest dimension and depth variation $0.5 \mathrm{~m}<\mathrm{d}<$ $2.0 \mathrm{~m}$, confirms the existence of mutual transmit-receive far-field propagation conditions over the system's complete bandwidth ( $~ 0.5$ to $2.5 \mathrm{GHz}$ ). Subsequent application of farfield Friis equation analysis [12], to the identical, transmit and receive ring monopoles with directivities $G_{T}\left(\theta_{n}, \omega\right)$ and $G_{R}\left(\theta_{n}, \omega\right)$, enables the power dissipated in the $n^{\text {th }}$ sensor load impedance $Z_{L}$, at displacement $d_{n}$ from the transmitter to be derived as :

$$
\frac{\left|S_{n}\left(\theta_{n}, \phi_{n}\right) S_{n}\left(\theta_{n}, \phi_{n}\right) *\right|}{Z_{L}}=\frac{P_{T} G_{T}\left(\theta_{n}, \phi_{n}, \omega\right) G_{R}\left(\theta_{n}, \phi_{n}, \omega\right)\left|\mathrm{e}^{-j \beta_{0} \sqrt{\varepsilon^{*}} d_{n}}\right|^{2}}{4 \pi d_{n}{ }^{2}}
$$

where $S_{n}\left(\theta_{n}, \phi_{n}\right)$ is the received signal strength at the $n^{\text {th }}$ antenna terminating impedance, $P_{T}$ is the transmit sensor input power and $\varepsilon^{*}$ is the complex permittivity of the wheat propagation medium. Assuming the antennas in Fig. 3 exhibit a directional dependence in the E-plane / $\theta$-plane and isotropic dependence in the $H$-plane/ $\phi$-plane, i.e $G_{T}\left(\phi_{n}\right)=1$, then the received signal reduces to :

$$
S_{n}\left(\theta_{n}\right)=\frac{A_{0}\left[F_{T}\left(\theta_{n}, \omega\right) F_{R}\left(\theta_{n}, \omega\right)\right]^{1 / 2} \mathrm{e}^{-j \beta_{0} \sqrt{\varepsilon^{*}} d_{n}}}{d_{n}} \cdot \sqrt{\frac{Z_{L}}{4 \pi}}
$$

where the antenna directivities in (3) are expressed in terms of E-plane bore sight gain and array factor via the relationship $G\left(\theta_{n}, \omega\right)=G(0, \omega) F\left(\theta_{n}, \omega\right)$ and the const. $A_{0}$ is given by:

$$
A_{0}=\sqrt{\frac{P_{T} G_{T}(0, \omega) G_{R}(0, \omega) Z_{L}}{4 \pi}}
$$

\section{B. The synthetic aperture sensor radiation pattern}

The wheat embedded sensor elements shown in Fig. 3 comprise novel UWB annular ring monopole antennas etched onto printed circuit board [10]. These are designed to exhibit uniformity of gain and linearity over a broad 0.5-2.5 GHz. bandwidth whilst displaying a 


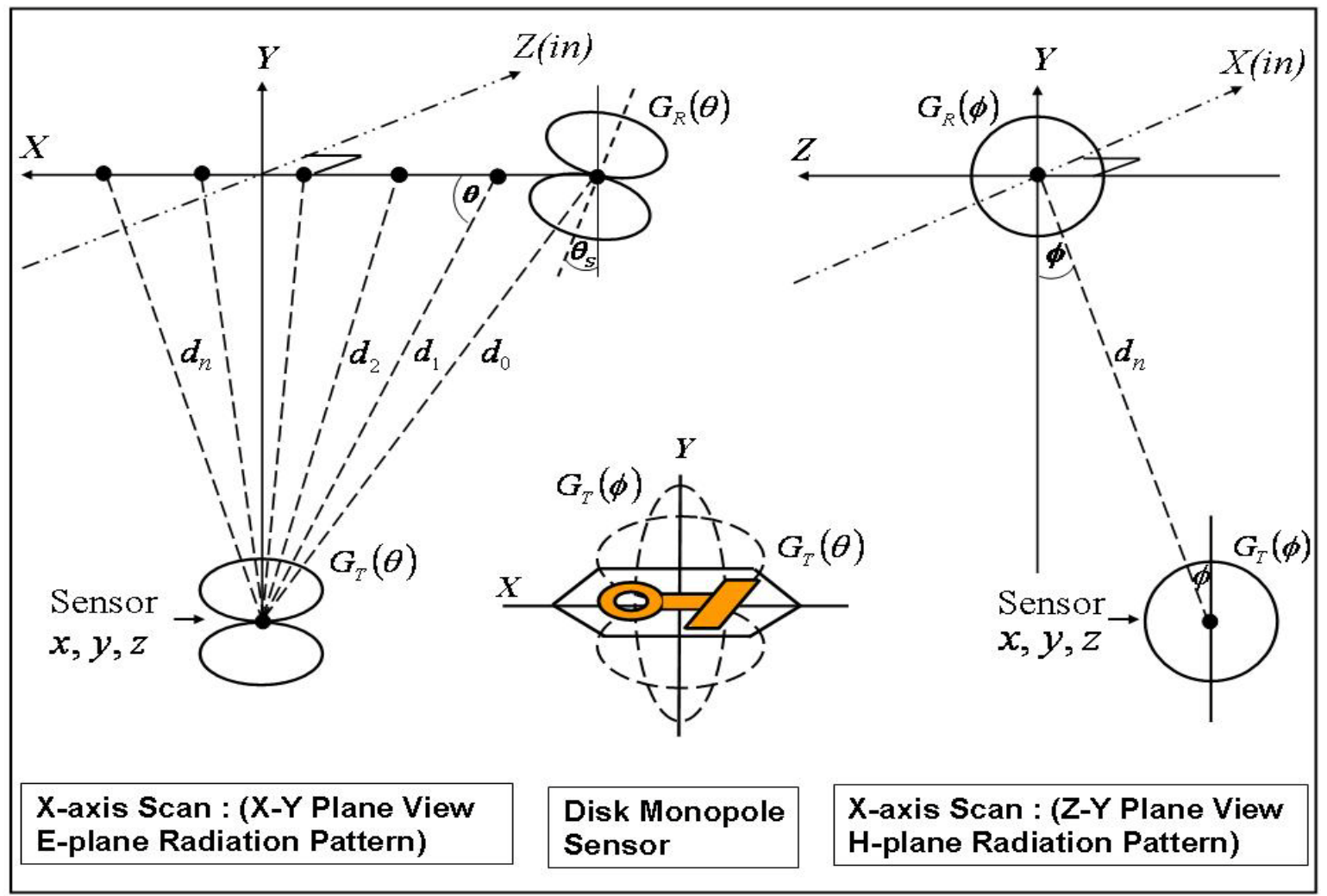

Figure $3: \mathrm{X}$ and $\mathrm{Z}$ axis 3-D synthetic aperture positioning configuration showing ring monopole antenna radiation pattern and bore sight 'squint' angle orientation.

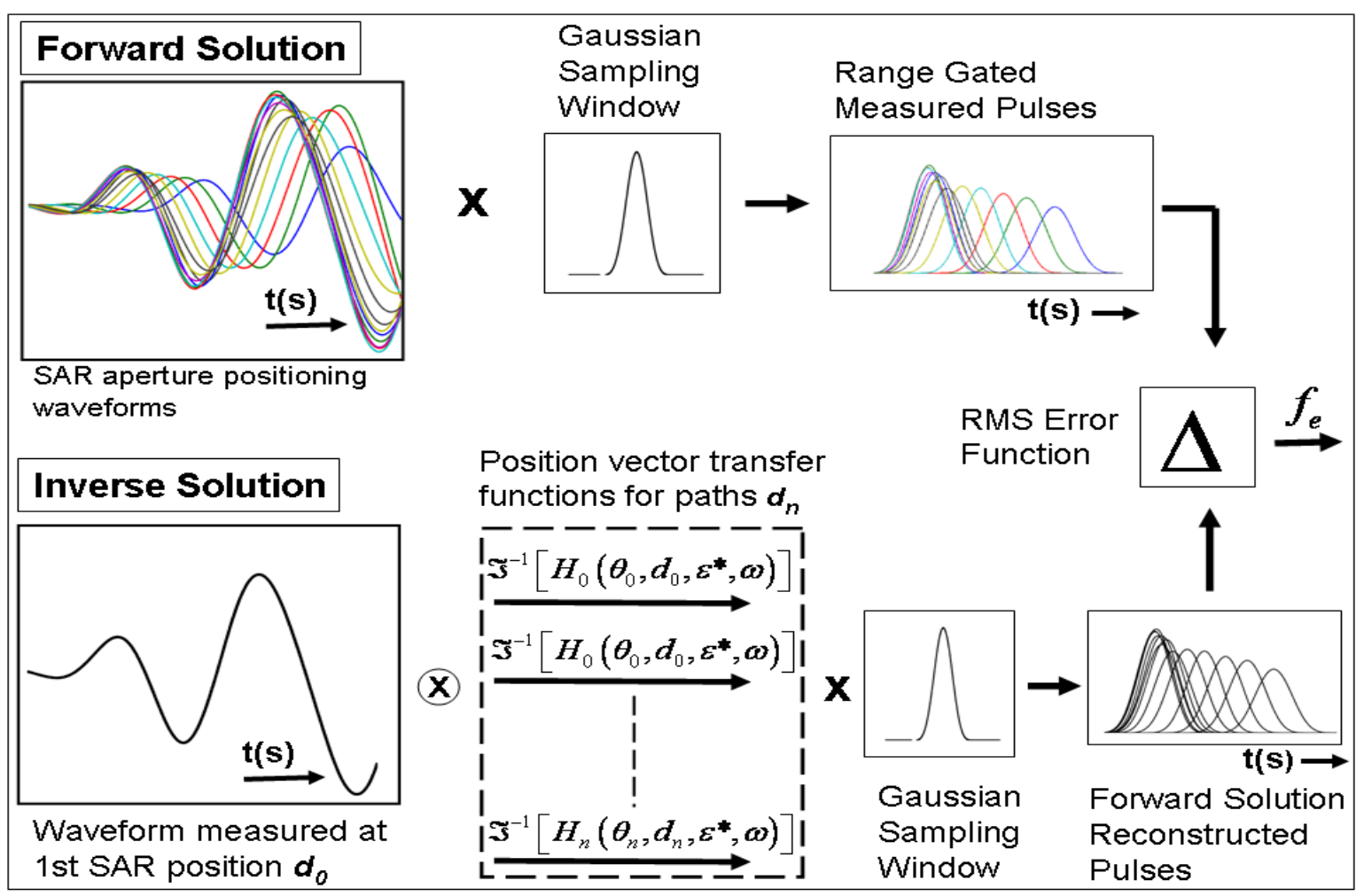

Fig. 4 . Overview of inversion methodology used to i) acquire wheat complex permittivity and sensor position data ii) minimise error between measured and reconstructed pulses. 
high degree of phase centre stability. The antennas exhibit a differentiating wideband output characteristic, whilst maintaining a high degree of pulse fidelity and modest return loss $(<2.2)$. Furthermore, their compact printed circuit board dimensions $(120 \mathrm{~mm} \times 75$ $\mathrm{mm}$ ) facilitate ease of operation as either synthetic aperture (SAR) receive rail receive sensors or embedded wheat transmitting elements. The radiation pattern over this frequency range exhibits a broader beam version of the 'classic' toroidal shaped array factor characteristic [13], normally associated with a monopole in elevation $(\theta)$ and azimuthal $(\phi)$ planes of the form:

$$
F_{T}(\theta)=\frac{\cos \left(\frac{\pi}{2} \cos (\theta)\right)}{\sin (\theta)} \approx \sin ^{k}(\theta) \quad ; \quad F_{T}(\phi)=1
$$

The broadening of the antenna beam pattern, directly attributable to the dispersive effect of the propagation medium, is a direct function of the wheat based permittivity and tends to reduce the index on the LHS in (5) to a value less than its free space equivalent of $k=3$, i.e $F\left(\theta_{n}\right) \approx \sin ^{3}\left(\theta_{n}\right)$. This value of $k$ is incorporated into the forward solution to reflect its part in determining the variation in SAR received pulse amplitude with elevation angle. Hence for identical antennas, where $F_{T}=F_{R}$, the expression of (3) simplifies to:

$$
S_{n}\left(\theta_{n}, \omega\right)=\frac{A_{0} \sin ^{k}\left(\theta_{n}-\theta_{s}\right) \mathrm{e}^{-j \beta_{0} \sqrt{\varepsilon^{*}} d_{n}}}{d_{n}} .
$$

where $\theta_{s}$ is the antenna squint angle in the antenna elevation plane and $\theta_{n}$ represents propagation vector elevation angle for the $n^{\text {th }}$ measurement position.

\section{Synthetic aperture array transfer function}

The overall receive aperture / emitter transfer function, which facilitates the prediction of the $m^{\text {th }}$ aperture received signal from that measured at the $n^{\text {th }}$, is defined from the ratio of received signal strengths at the $m^{\text {th }}$ and $n^{\text {th }}$ positions, which may be derived from (6) as: 


$$
H_{m n}(\omega)=\frac{S_{m}\left(\theta_{m}, \omega\right)}{S_{n}\left(\theta_{n}, \omega\right)}=\left(\frac{d_{n}}{d_{m}}\right)\left[\frac{\sin \left(\theta_{m}-\theta_{s}\right)}{\sin \left(\theta_{n}-\theta_{s}\right)}\right]^{k} \mathrm{e}_{\sqrt{ }}^{-j \beta_{0} \sqrt{\varepsilon^{*}}\left(d_{m}-d_{n}\right)}
$$

where the radial position vectors $d_{n}$ for $n^{\text {th }}$ measurement position, may be expressed in terms of sensor coordinates $x, y, z$ and receiving sensor separation $L_{x}$ and $L_{z}$ for each of the $x$ and $z$ axis scans as :

$$
d_{n}^{x}=\left[\left(x-n L_{x}\right)^{2}+y^{2}+\left(z-z_{0}\right)^{2}\right]^{1 / 2} ; d_{n}^{x}=\left[\left(x-n L_{x}\right)^{2}+y^{2}+\left(z-z_{0}\right)^{2}\right]^{1 / 2}
$$

and the inclination angles of the positioning vectors with respect to their respective $x \& z$ axes are given by :

$$
\theta_{n}^{x}=\cos ^{-1}\left(\frac{x-n L_{x}}{d_{n}^{x}}\right) \quad ; \quad \theta_{n}^{z}=\cos ^{-1}\left(\frac{z-n L_{z}}{d_{n}^{z}}\right)
$$

In essence, the function defined in (7) now represents the inversion algorithm forward solution, with a set of optimisation variables comprising the 3-D sensor position coordinates, wheat complex permittivity $\varepsilon^{*}$ and antenna 'correction factor' $k$.

\section{System propagation delay and impulse response}

Both approximate and rigorous Gaussian pulse reconstruction estimates for complex permittivity and emitter position coordinates, require knowledge of the system impulse response in order that the amplitude, propagation delay and location of the pulse maxima be calculated. This may be derived via the IFFT of the pulse transfer function in (7) to yield the received signal time dependence at the $m^{\text {th }}$ SAR array position as :

$$
S_{m}(t)=\mathfrak{J}^{-1}\left[S_{n}(\omega) \cdot H_{m n}(\omega)\right]=S_{n}(t) \otimes h(t) \otimes \delta(t-\Delta t)
$$

from which the normalised impulse response amplitude $h(t)$ is given by : 


$$
h(t)=\left(\frac{d_{n}}{d_{m}}\right)\left[\frac{\sin \left(\theta_{m}-\theta_{s}\right)}{\sin \left(\theta_{n}-\theta_{s}\right)}\right]^{k} \mathfrak{J}^{-1}\left[e^{\beta_{0} \sqrt{\mathcal{E}^{*}} \sin \left(\frac{\delta}{2}\right)\left(d_{m}-d_{n}\right)}\right]
$$

and the pulse maxima propagation delay $\Delta t$ is defined as :

$$
\Delta t=\frac{\sqrt{\left|\varepsilon^{*}\right|} \cos (\delta / 2)\left(d_{m}-d_{n}\right)}{c_{0}}
$$

where $\left|\varepsilon^{*}\right|=\sqrt{\left(\varepsilon^{\prime}\right)^{2}+\left(\varepsilon^{\prime \prime}\right)^{2}}$ is the complex permittivity modulus, $\delta=\tan ^{-1}\left(\varepsilon^{\prime \prime} / \varepsilon^{\prime}\right)$ the loss tangent, and $d_{n}$ and $d_{m}$ the propagation path lengths defined in (8). Subsequent geometric analysis of the displacement and orientation of transmit and receive sensors from (8), along with manipulation of (12), enables the classic hyperbolic dependence between $x$ or z-axis receive pulse position and propagation delay to be established. Hence for the $z$-axis case:

$$
\left(\frac{\Delta t_{z}}{A_{z}}\right)^{2}-\left(\frac{z-n L_{z}}{B_{z}}\right)^{2}=1
$$

where $A_{z}= \pm p \sqrt{\left(x-x_{0}\right)^{2}+y^{2}} ; \quad B_{z}= \pm A_{z} / p \quad$ and $\quad p=\left(\sqrt{\left|\varepsilon^{*}\right|} / c_{0}\right) \cos (\delta / 2) \cdot \mathrm{A}$ comparison of the measured and reconstructed pulse maxima at each array location in Figs. 5(a) and 5(b), reveals the hyperbolic delay locus of (13), indicated by the dashed line. This along with more detailed scrutiny of measured and optimised pulse waveforms and subsequent discussion of the correlation between the amplitude function (11) and pulse maxima values are provided in Section 5 below.

\section{E. Down and Cross range Resolution}

Establishing the fundamental relations for the down and cross range resolution of the synthetic aperture positioning system, is a fundamental pre-requisite for accurate sensor detection in the spatial domain $[14,15]$. For example, in the vertical or down-range dimension, where the resolving capability is dictated by the width of the Gaussian pulse 
received at the aperture, the resolution is determined by the pulse bandwidth $\Delta f$, host medium permittivity $\varepsilon^{*}$ and loss tangent $\delta$ via the relation:

$$
\Delta y \approx c_{0} /\left(2 \Delta f \sqrt{\left|\varepsilon^{*}\right|} \cos (\delta / 2) e^{-\alpha y}\right)
$$

where the propagation decay constant $\alpha=\beta_{0} \sqrt{\left|\varepsilon^{*}\right|} \sin (\delta / 2) \mathrm{m}^{-1}$, governs the deterioration in resolution with depth $y / m$ due to signal dispersion. The cross-range resolution [16], is determined by the receive aperture principal lobe width in Fig 3, which to a first approximation is given by:

$$
\Delta x \approx y \lambda /\left(D \sqrt{\left|\varepsilon^{*}\right|} \cos (\delta / 2)\right)
$$

where $D=n L_{x}$ is the effective aperture length for $n$ measurement positions and interelement spacing $L_{x}$. Subsequent application of (14) \& (15) to the measurement configuration of Fig. 3, for aperture length $\mathrm{D}=1.0 \mathrm{~m}, \varepsilon^{\prime}=2.7, \varepsilon^{\prime \prime}=0.1 ; \Delta f=2.5 \mathrm{GHz}$, yields a cross range resolution of $5.8 \mathrm{~cm}$ at the $1.0 \mathrm{GHz}$ mid-band frequency, with a down range resolution varying from $3.5 \mathrm{~cm}$ at the surface to $6.2 \mathrm{~cm}$ at a $1.0 \mathrm{~m}$ depth. This depth dependent deterioration in resolving power, emphasises the need for customised weighting of the incident pulse power spectral density in order to minimise high frequency component loss and preserve positioning accuracy [17].

\section{The Inversion Algorithm}

Following on from the forward solution derivation, the complete inversion methodology, illustrated schematically in the process flow diagram of Fig. 4, yields the optimum set of transfer functions $H_{m n}^{x}(\omega)$ and $H_{m n}^{z}(\omega)$, for both $x$ and $z$ scan aperture axes, which best replicate the relative attenuation and propagation delay between the $n^{\text {th }}$ and $m^{\text {th }}$ measured pulses at the respective positioning array scanning positions. The 'correct' set of transfer functions will generate the global minimum difference between measured and reconstructed pulses, as derived from the IFFT of the overall system transfer function in (10), for all time values and positions, i.e 


$$
S_{m}^{x}(t)=\mathfrak{I}^{-1}\left[S_{n}^{x}(\omega) \cdot H_{m n}^{x}(\omega)\right] \quad ; \quad S_{m}^{z}(t)=\mathfrak{I}^{-1}\left[S_{n}^{z}(\omega) \cdot H_{m n}^{z}(\omega)\right]
$$

where $S_{m}^{x}(\omega)$ and $S_{m}^{z}(\omega)$ represent the measured oscilloscope signal strengths at the $m^{\text {th }}$ measurement position and $S_{n}^{x}(\omega)$ and $S_{n}^{z}(\omega)$ their counterparts at the $n^{\text {th }}$. The inversion error function for a given parameter space, commonly referred to as the optimisation algorithm 'forward solution', is derived from the mean square difference between the measured and reconstructed pulses for all positions and time values $t$ as :

$$
f(\bar{\zeta})=\frac{2}{N(N-1)} \sum_{n=1}^{N} \sum_{m>n}^{M}\left\{\left|S_{m}^{x}(t)-\mathfrak{J}^{-1}\left[S_{n}^{x}(\omega) \cdot H_{m n}^{x}(\omega)\right]\right|^{2}+\left|S_{m}^{z}(t)-\mathfrak{J}^{-1}\left[S_{n}^{z}(\omega) \cdot H_{m n}^{z}(\omega)\right]\right|^{2}\right\}^{1 / 2}
$$

where $N$ is the number of both the $x$ and $z$-axis measurement positions and $m>n$ for all $\mathrm{m}$. The optimisation parameter set over which the above function is minimised is defined as:

$$
\bar{\zeta}=\left[x, y, z, \varepsilon^{\prime}, \varepsilon^{\prime \prime}, k\right]
$$

where $x, y, z$ are the sensor position coordinates, $\varepsilon^{\prime}$ and $\varepsilon^{\prime \prime}$ the permittivity real and imaginary parts and ' $k$ ' the antenna pattern correction factor. The global solution to the inversion process yields a unique solution for the reconstructed parameter set $\bar{\zeta}$, such that little or no discernable difference between the measured and reconstructed data values will be revealed on comparison between pulse profiles. The inversion process itself comprises a search for the global minimum value of the function $f(\bar{\zeta})$, via application of a 'Minimum level single linkage algorithm' [18] to the measured SAR positioning data over a predefined feasible range. The parameter set corresponding to this minima, offers a distinct solution identifying the optimum 'estimate' of sensor coordinates, radiation pattern correction factor and complex permittivity. The aforementioned optimisation 'feasible range', specified in Table 1 , specifies the extent over which the $P$ components of $\bar{\zeta}$ vary and effectively limits the size of the $P$-dimensional parameter space in which the global minima may be located. Sensible limitations on this range are an important consideration for the relatively large $(P=6)$ values encountered here, as these affect the overall computational efficiency of the optimisation process. 
Table 1: Optimisation Parameter Feasible Range

\begin{tabular}{|c|c|c|c|c|c|}
\hline $\mathrm{X}(\mathrm{m})$ & $\mathrm{Y}(\mathrm{m})$ & $\mathrm{Z}(\mathrm{m})$ & $\varepsilon^{\prime}$ & $\varepsilon^{\prime \prime}$ & $k$ \\
\hline 0 to 1.0 & 0 to 2.0 & 0 to 1.0 & 1.0 to 4.0 & 0.0 to 0.1 & 1.0 to 4.0 \\
\hline
\end{tabular}

\section{Optimisation Analysis and Results}

In this chapter, the accuracy of the inversion algorithm positioning and permittivity estimates are compared for a single transmitting source at various positions in a 3-D volume within the plastic vessel, via measurement of the time difference of arrival data at 5 cm intervals on both the $X$ and $Z$ receiving aperture as shown in Fig. 3. Typical time domain profiles of the form shown in Fig. 2 were considered on each axis for 4 test positions in total. Note, a detailed overview of this complete inversion methodology is illustrated schematically in Fig. 4.

After pre-processing with the range gate to isolate the line of sight component, the inversion algorithm, yielded the optimum sensor position coordinates, complex permittivity and antenna pattern correction factor required to minimise the difference between measured and reconstructed data. The four selected test positions were chosen to provide a reasonable spread in the severity of multi-path interference within the confines of the plastic vessel. Evidence of the degree of fidelity of the sensor position and complex permittivity inversion estimates may be gleaned via either an approximate method based on the evaluation of the reconstructed pulse peak amplitude and delay as provided in sections $\mathrm{A}$ and $\mathrm{B}$, or a more rigorous approach comprising a reconstructed pulse parameter assessment for all time domain values in section $\mathrm{C}$ and $\mathrm{D}$. Both techniques are considered starting with the approximate pulse peak delay / amplitude comparison first where the fidelity of the reconstruction is assessed graphically via a comparison of the measured and optimised propagation delay and amplitude estimates. Section E, which provides an assessment of the accuracy of positioning coordinates and associated permittivity data follows on from this.

\section{A. Comparison of measured and reconstructed pulse propagation delays}

Firstly, the accuracy of the reconstructed pulse propagation delays for the optimised permittivity and emitter position coordinates, are assessed for the single wireless emitter test position 2, to provide greater insight into the fidelity of pulse reconstruction 
methodology within dispersive media. Here, application of the global solution parameters to the propagation path impulse function (11) enables the pulse profiles for both $X$ and $Z$ synthetic aperture positioning axes to be reconstructed for all $N$ array positions. These reconstructed pulses are plotted against their measured $X$ and $Z$ axis pulse counterparts in Figs. 5(a) and 5(b) respectively, where the ordinate represents the SAR axis receive position and the measured pulse peak values are denoted by a series of crosses. The locus of the theoretical propagation delay, attributable to the Gaussian pulse maxima for $X$ and $Z$ axis synthetic aperture measurements, has been derived from the impulse delay function in (12) and is superimposed onto the respective Figs. 5(a) \& (b). A study of these composite plots, reveals how the prescribed theoretical hyperbolic loci, on passing through the pulse peak values in each illustration, yield a clear indication that the inversion parameter estimate for this particular propagation scenario (i.e $x=0.443, y=0.927, z=0.545, \varepsilon^{\prime}=$ 2.408, $\varepsilon^{\prime \prime}=0.0293$ ), provides a realistic evaluation of the peak pulse propagation delay. This result is further verified via a study of the pulse 'error' function illustrated for $\mathrm{X}$ and $\mathrm{Z}$ axes in Figures 6(a) and 6(b) respectively, where the normalised difference between measured and reconstructed pulses is shown not to exceed $15 \%$ of the peak value in either instance.

The correlation between both measured and reconstructed pulse peak propagation delays with their theoretical counterparts is further scrutinised in Figures 7(a) and 7(b) for both $X$ and Z-axes respectively. Here curves derived via interpolation between the maxima of i) the measured pulse profiles ii) inversion or reconstructed pulse profiles and iii) the theoretical propagation delay function (13) are plotted on the same axes. Note the close proximity of the theoretical and inversion interpolation curves which correlate to within $10 \%$ of the measured profile over the 0 to $0.60 \mathrm{~m}$ long Z-axis scan aperture. Any discrepancy between these may be attributed to the origin of the reconstructed profiles in Figures 5(a) \& 5(b), where the inversion fit is derived via minimisation of both amplitude and propagation delay values for all discrete time domain points, as opposed to the theoretical hyperbolic function (13) which endeavours to provide a 'best fit' for the peak pulse propagation delay only. 


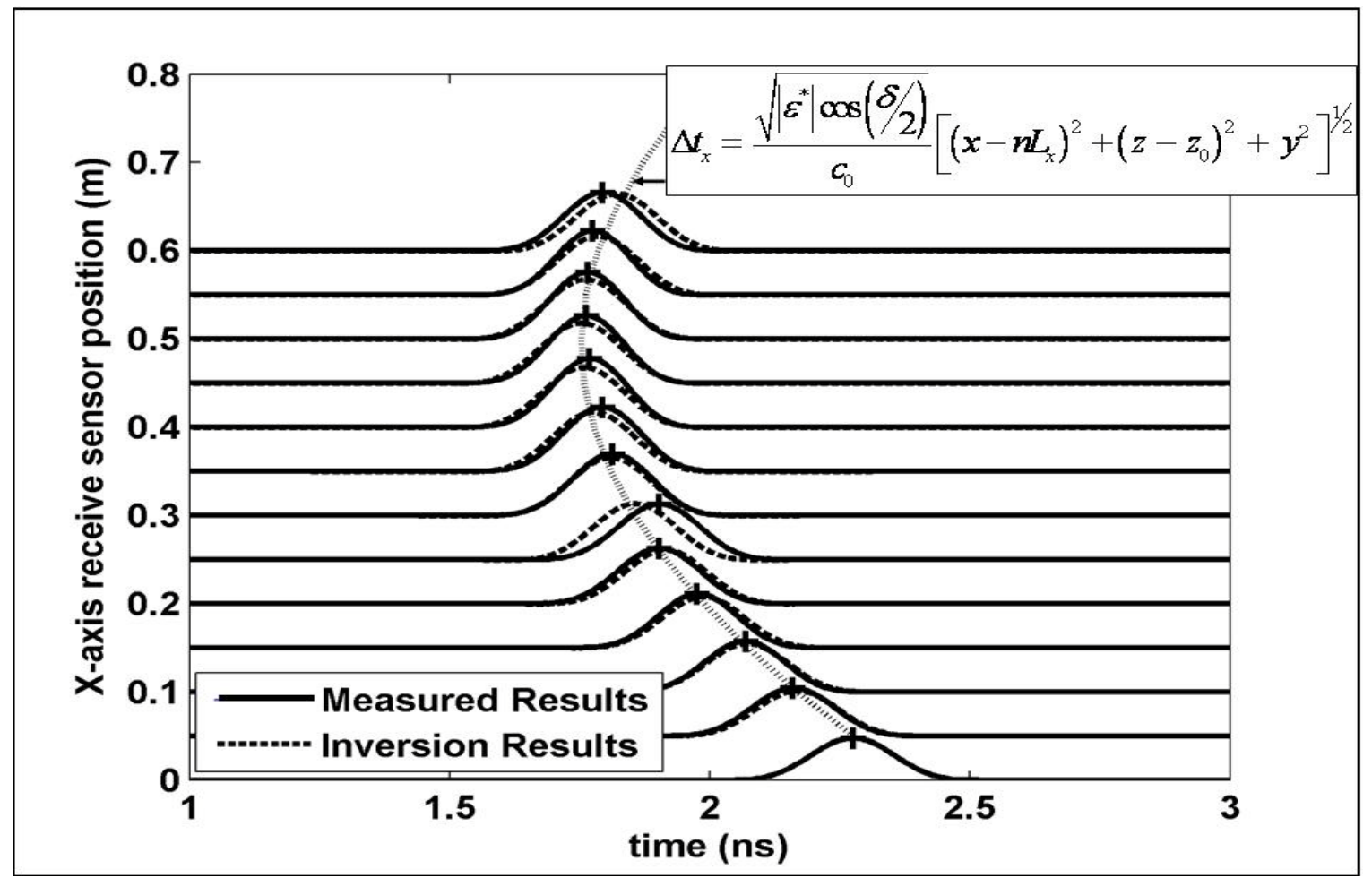

Figure 5(a) : X-axis measured \& inversion algorithm 'best fit' time domain responses of ring monopole antennas in wheat for monocycle pulse excitation after Gaussian range gate filtering for receive antenna measurement positions $X_{n}=0,5,10,15, \ldots 60 \mathrm{~cm}$ at Test Position 2.

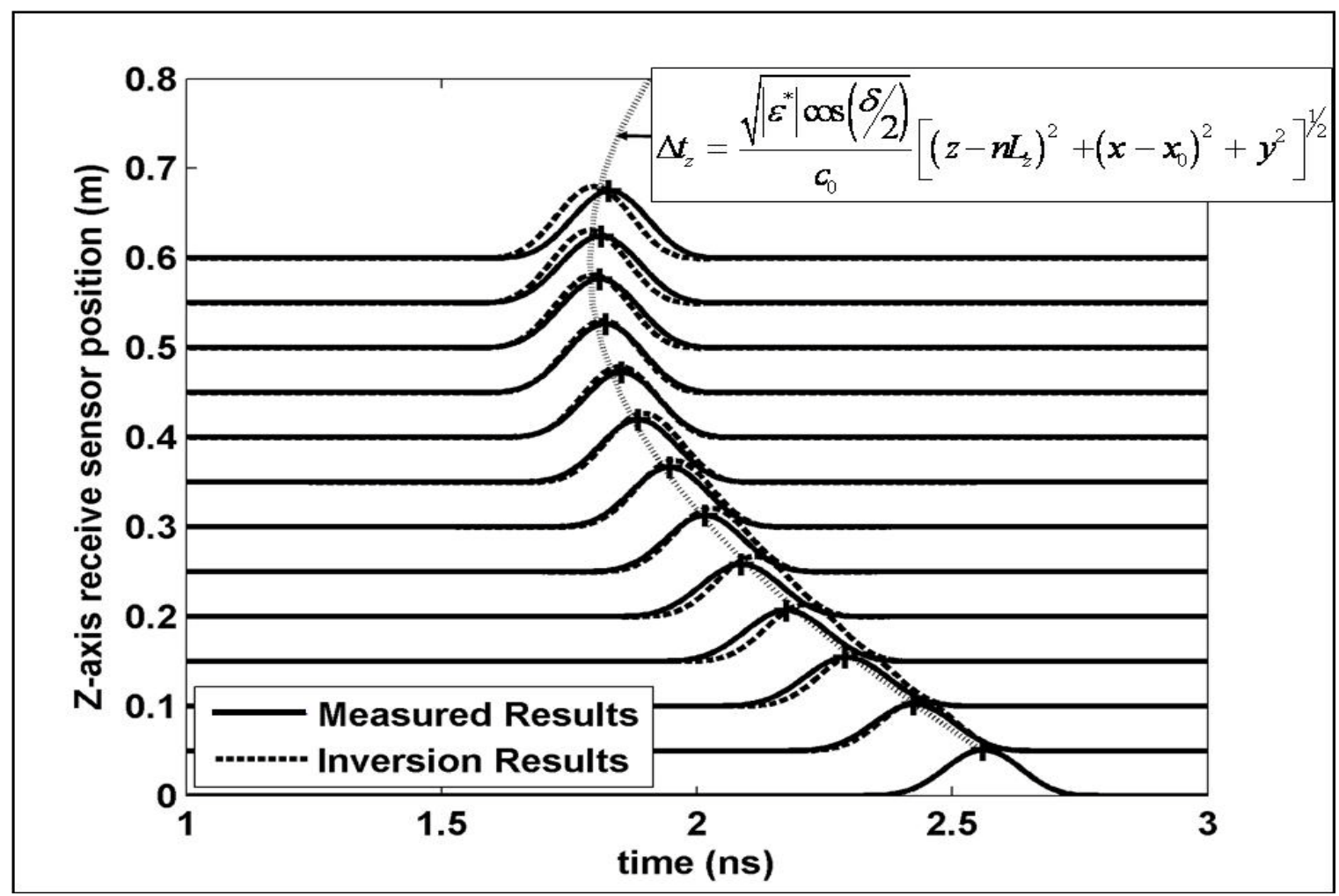

Figure 5(b) : Z-axis measured \& inversion algorithm 'best fit' time domain responses of ring monopole antennas in wheat for monocycle pulse excitation after Gaussian range gate filtering for receive antenna measurement positions $X_{n}=0,5,10,15, \ldots 60 \mathrm{~cm}$ at Test Position 2 . 


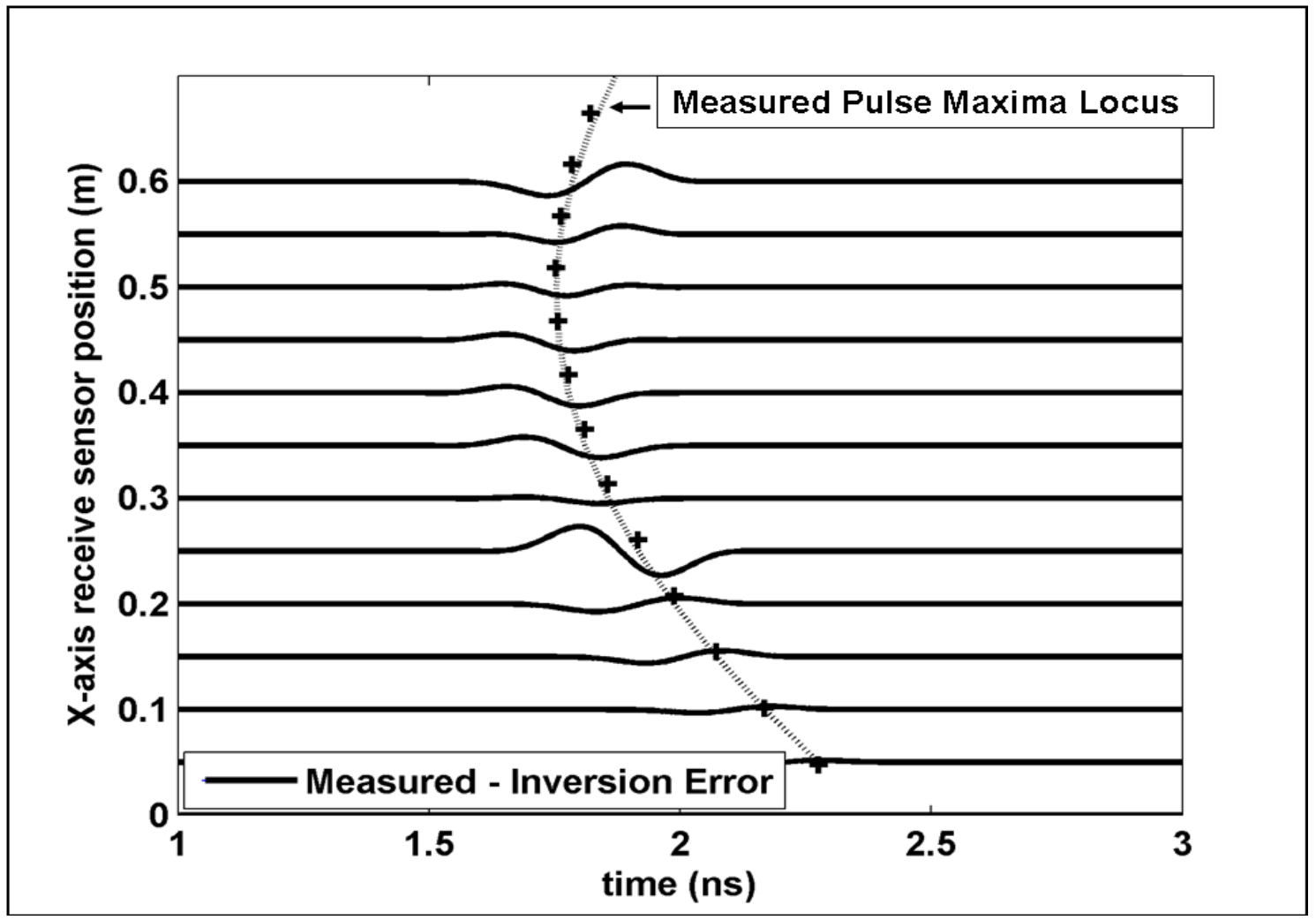

Figure 6(a) : X-axis difference between measured and inversion or 'best fit' time domain reconstructed responses shown in Figures 5(a).

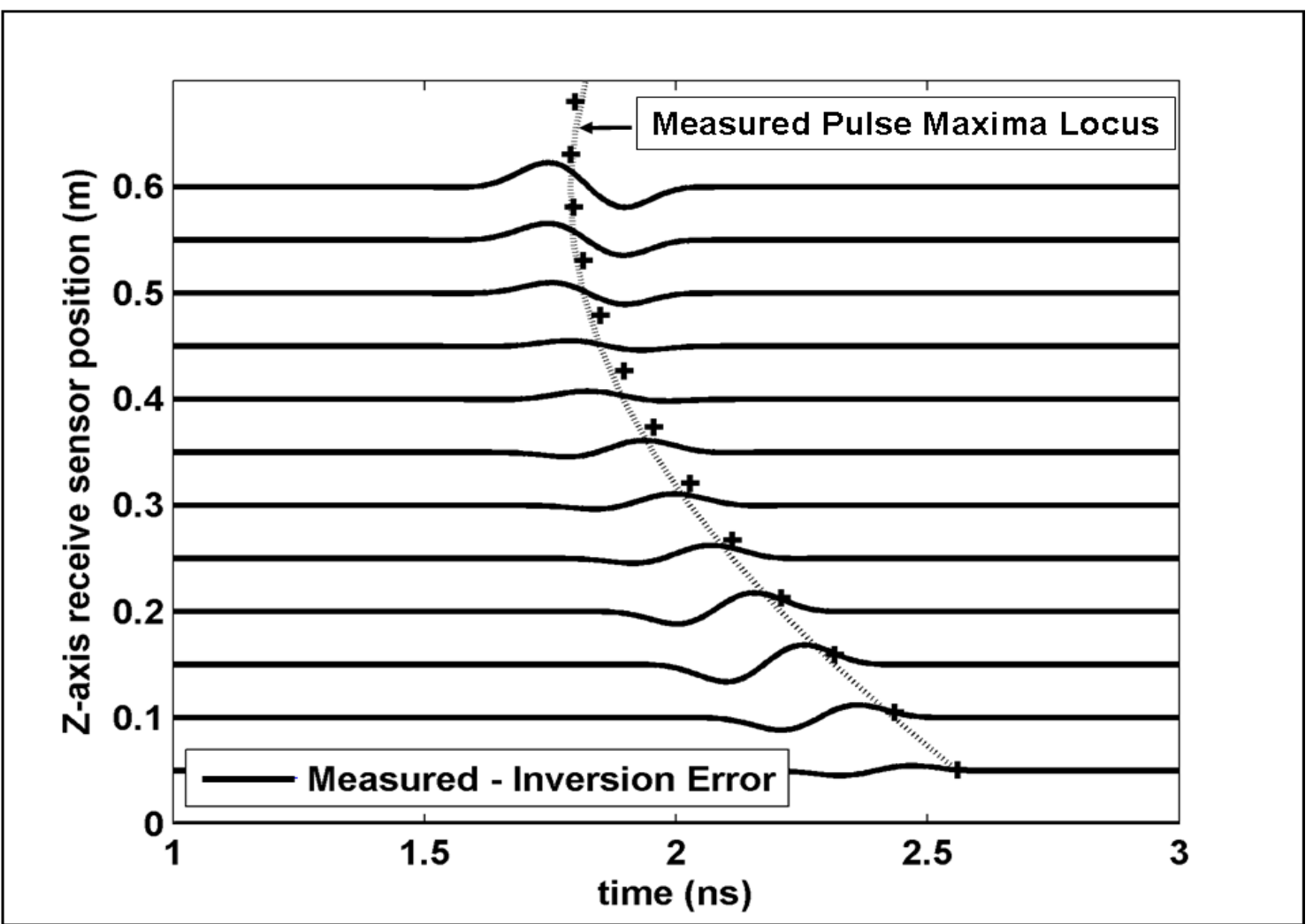

Figure 6(b) : Z-axis difference between measured and inversion or 'best fit' time domain reconstructed responses shown in Figures 5(b). 


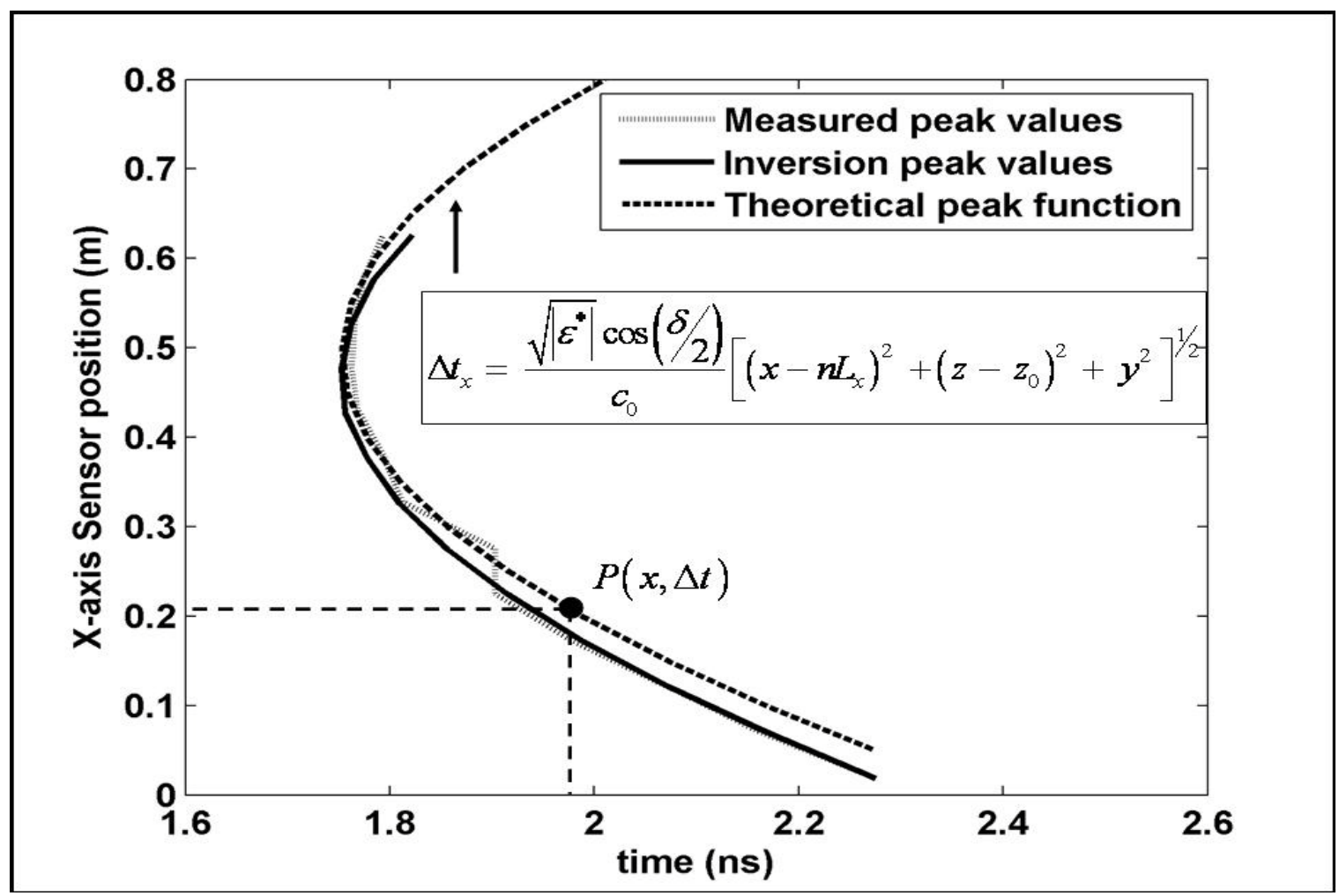

Fig. 7(a) : Schematic showing hyperbolic dependence of pulse peak propagation delay on $\mathrm{X}$-axis synthetic aperture position for measured, reconstructed and theoretical functions at Test Position 2.

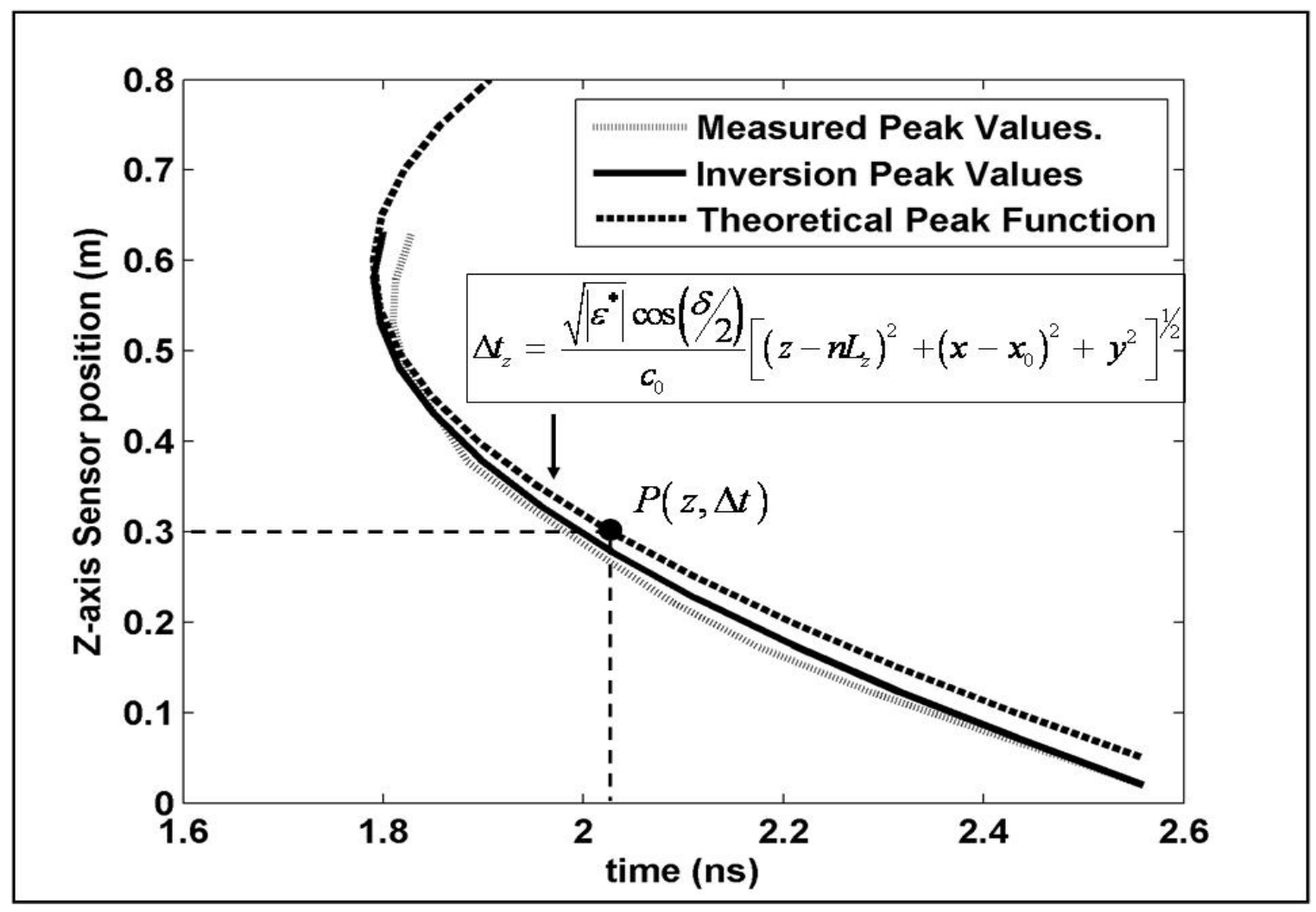

Fig. 7(b) : Schematic showing hyperbolic dependence of pulse peak propagation delay on Z-axis synthetic aperture position for measured, reconstructed and theoretical functions at Test Position 2. 


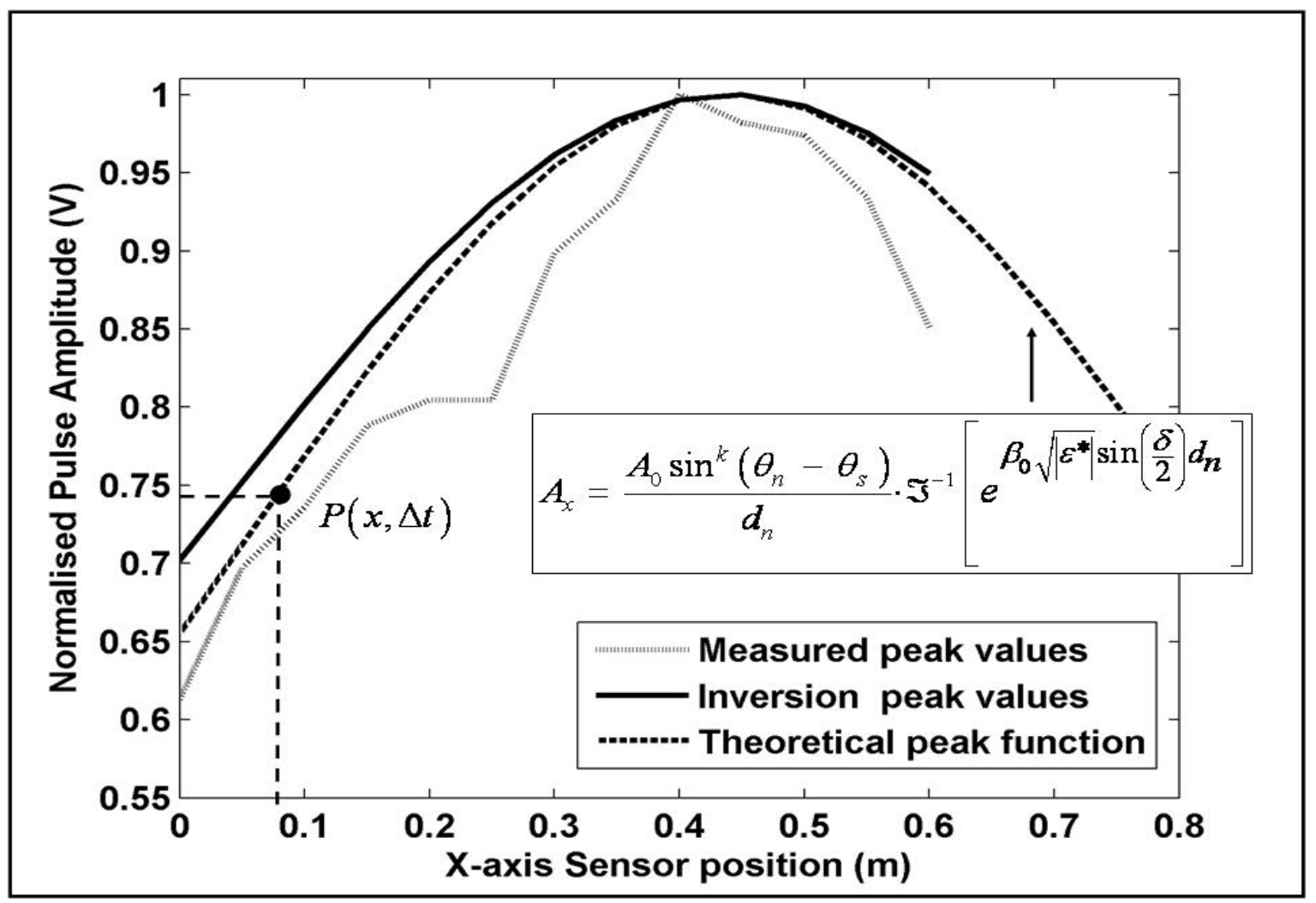

Fig. 8(a) : Schematic showing hyperbolic dependence of pulse peak amplitude on $\mathrm{X}$-axis synthetic aperture position for measured, reconstructed and theoretical functions at Test Position 2.

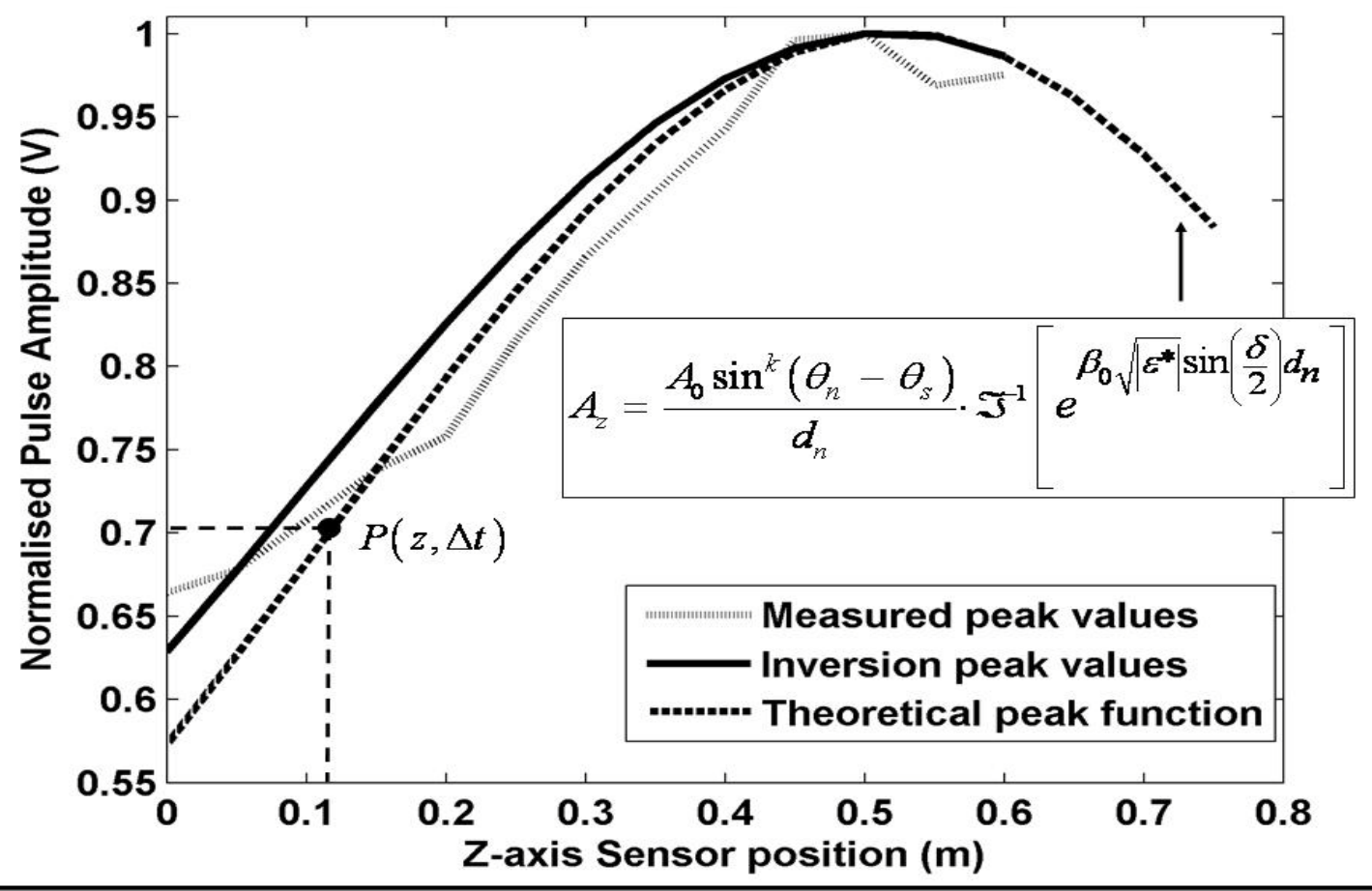

Fig. 8(b) : Schematic showing hyperbolic dependence of pulse peak amplitude on Z-axis synthetic aperture position for measured, reconstructed and theoretical functions at Test Position 2. 


\section{B. Comparison of measured and reconstructed pulse amplitudes}

Next, the approximate pulse comparison method is applied to both measured and reconstructed pulse peak amplitudes in Figures 8(a) and (b), where the abscissa represents the respective axis receive position and the ordinate the amplitude. Here loci derived via interpolation between the pulse maxima at test position 2, are superimposed on to the theoretical counterpart derived using the impulse function (11) for $X$ and $Z$ axis SAR measurements respectively. Note, the coincidence of the hyperbolic function turning points, with those exhibited in the propagation delay plots of Figs $7(a)$ and 7(b) and the actual sensor $X$ and $Z$ axis coordinate positions in Table 2, (i.e, $x \sim 0.45 \mathrm{~m}, z \sim 0.55 \mathrm{~m}$ ). Closer scrutiny of these measured and reconstructed peak amplitude functions reveals a degree of correlation not quite as close as that exhibited in the propagation delay analyses of Sections $A \& B$. The set of differences here may be principally attributed to positional errors in the mechanical support structure, the unknown antenna boresight squint offsets $\theta_{s}^{z}$ and $\theta_{s}^{x}$ defined in (9) and the inherent overall greater susceptibility of pulse amplitude estimates to material loss tangent variations [22].

\section{Rigorous comparison of measured and reconstructed pulses}

In this section a more substantive analysis of the inversion process is undertaken via comparison of measured and reconstructed pulse data amplitudes for all sampled time values as opposed to the peak value based assessment undertaken in the previous section $B$. In essence, this more rigorous approach simultaneously compares the amplitude and delay of the $\mathrm{X}$ and $\mathrm{Z}$-axis measured time domain responses for all receive-antenna positions $Z_{n}=$ $0,5,10 \ldots 60 \mathrm{~cm}$. The synthetic aperture array plots used for this purpose, now illustrate both measured and reconstructed pulses in 3-D form, with the ordinate representing pulse amplitude, the abscissa the pulse propagation delay and the normal Z-axis the synthetic aperture position. These plot profiles are compared for all four $a$ to $d$ test positions in Figures 9 to 12, with Figures 9(a-d) and 11(a-d) illustrating the measured pulses for $X$ and $\mathrm{Z}$ aperture axes respectively and Figures 10(a-d) and 12(a-d) their reconstructed counterparts. 


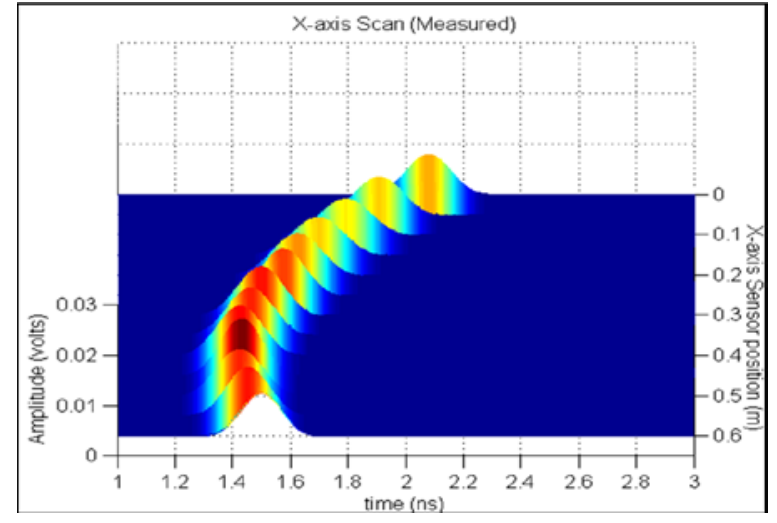

Fig 9(a) : 3D representation of $\mathrm{X}$-axis SAR aperture measured response after range gate filtering for test posn. 1.

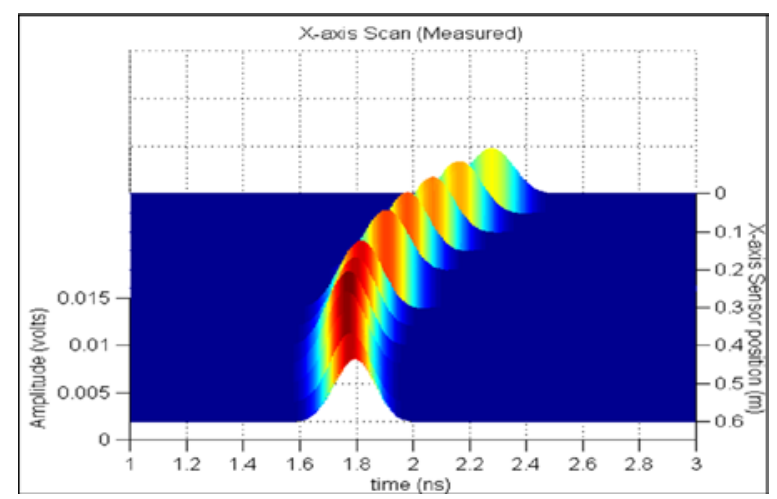

Fig 9(b) : 3D representation of $\mathrm{X}$-axis SAR aperture measured response after range gate filtering for test posn. 2 .

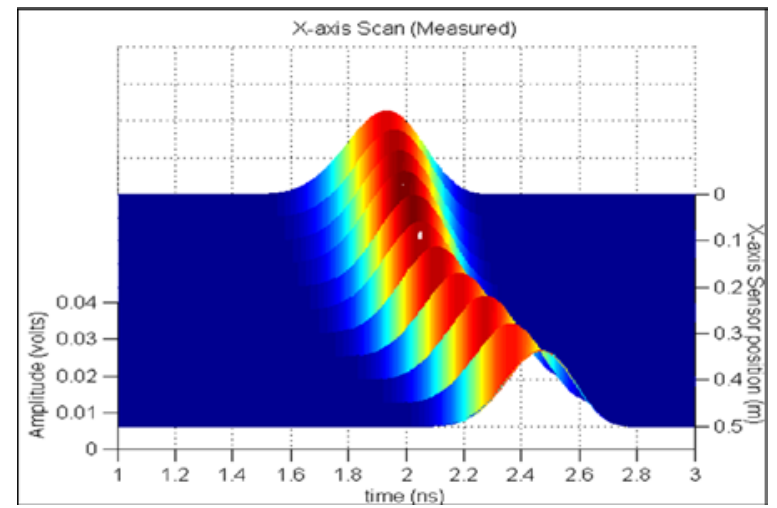

Fig 9(c) : 3D representation of $\mathrm{X}$-axis $\mathrm{SAR}$ aperture measured response after range gate filtering for test posn. 3 .

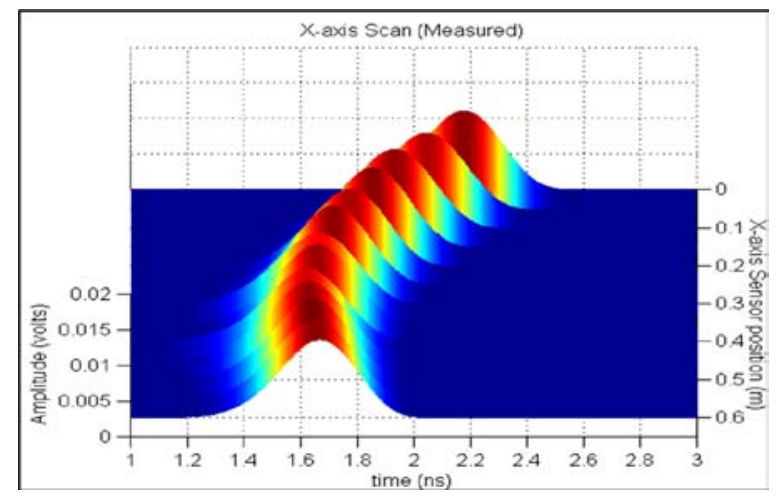

Fig 9(d) : 3D representation of $X$-axis SAR aperture measured response after range gate filtering for test posn. 4 .

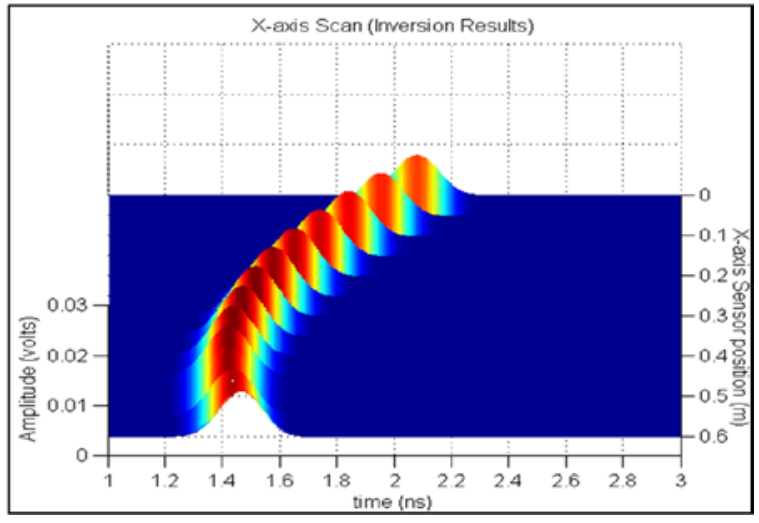

Fig 10(a) :. 3D representation of $X$-axis SAR reconstructed response after range gate filtering for test posn. 1 .

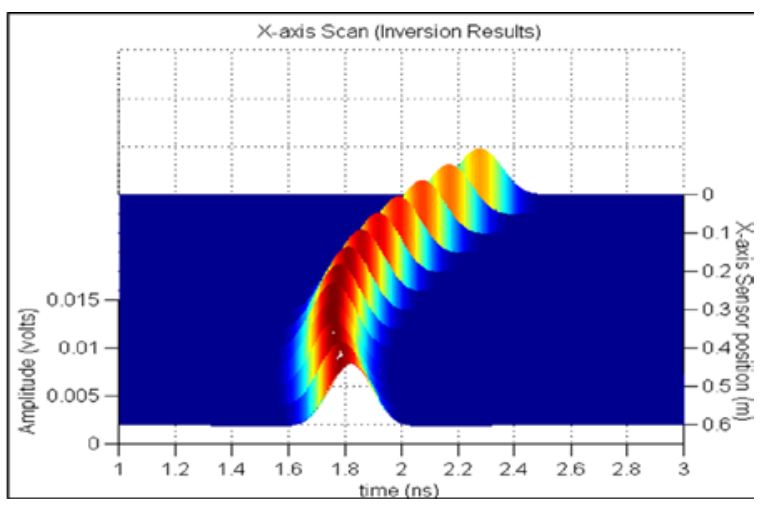

Fig 10(b) :: 3D representation of $X$-axis SAR reconstructed response after range gate filtering for test posn. 2 .

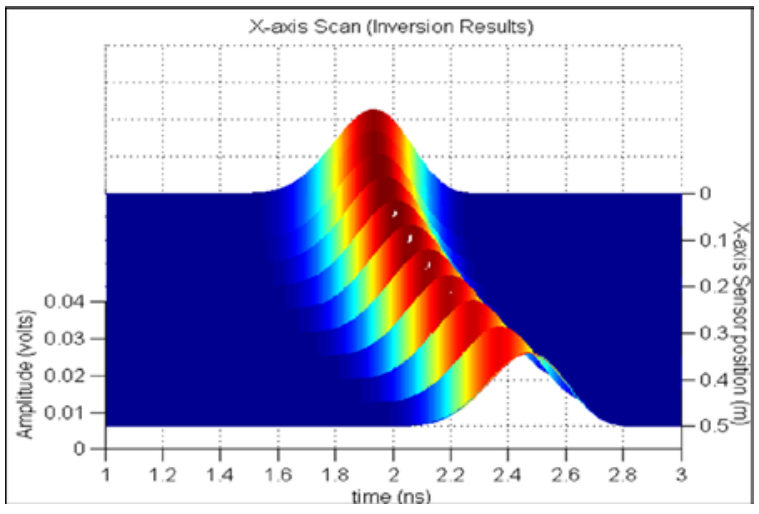

Fig 10(c) :. 3D representation of $\mathrm{X}$-axis SAR reconstructed response after range gate filtering for test posn. 3 .

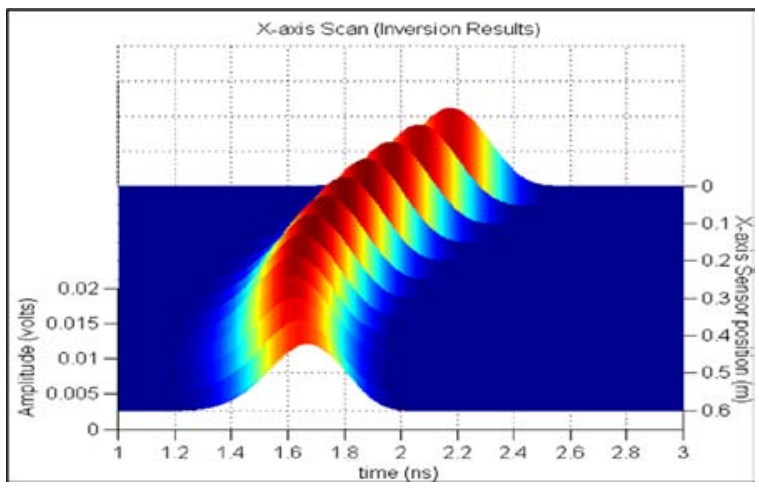

Fig 10(d) :. 3D representation of $X$-axis SAR reconstructed response after range gate filtering for test posn. 4 . 


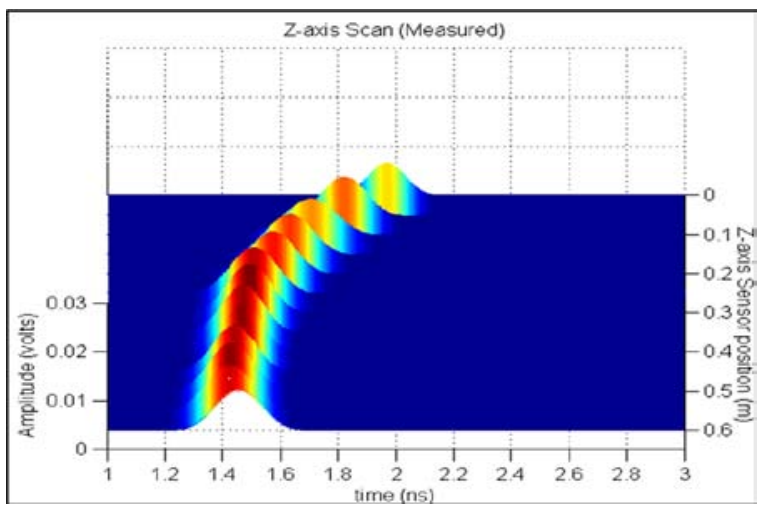

Fig 11(a) : 3D representation of $\mathrm{Z}$-axis SAR aperture measured response after range gate filtering for test posn. 1.

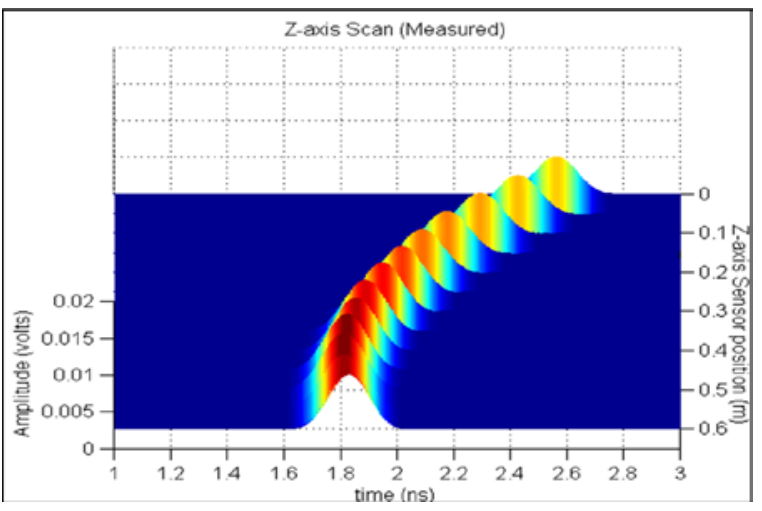

Figure 11(b) : 3D representation of Z-axis SAR aperture measured response after range gate filtering for test posn. 2 .

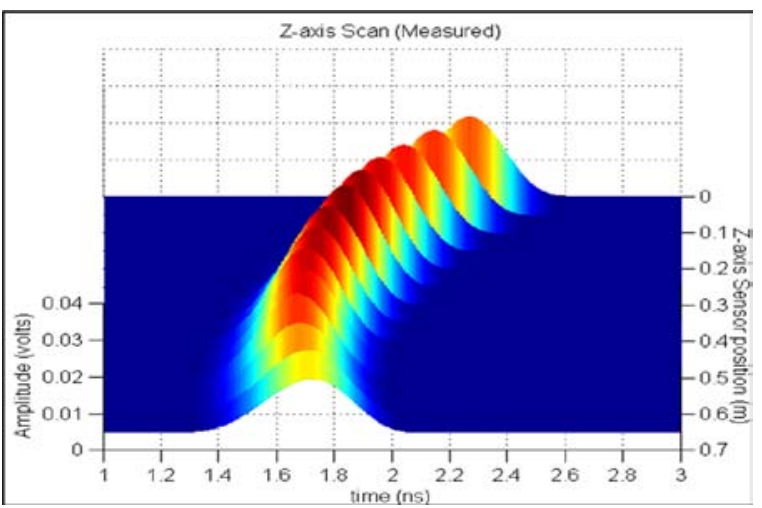

Figure 11(c) : 3D representation of Z-axis SAR aperture measured response after range gate filtering for test posn. 3 .

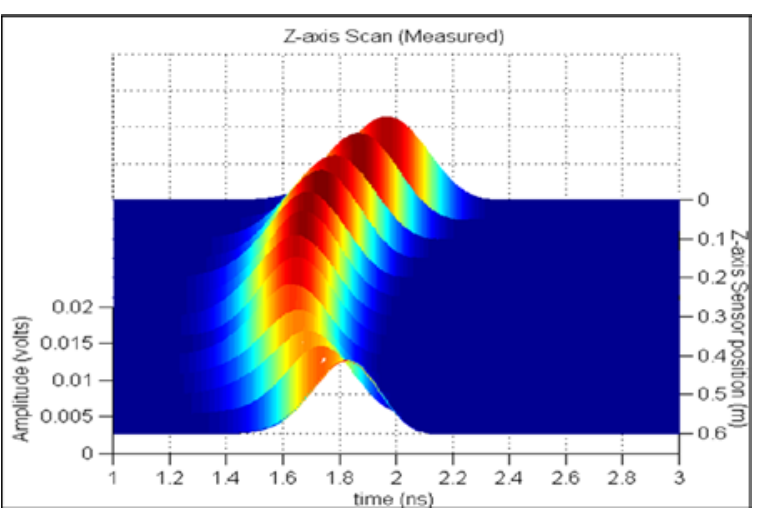

Figure 11(d) : 3D representation of Z-axis SAR aperture measured response after range gate filtering for test posn. 4 .

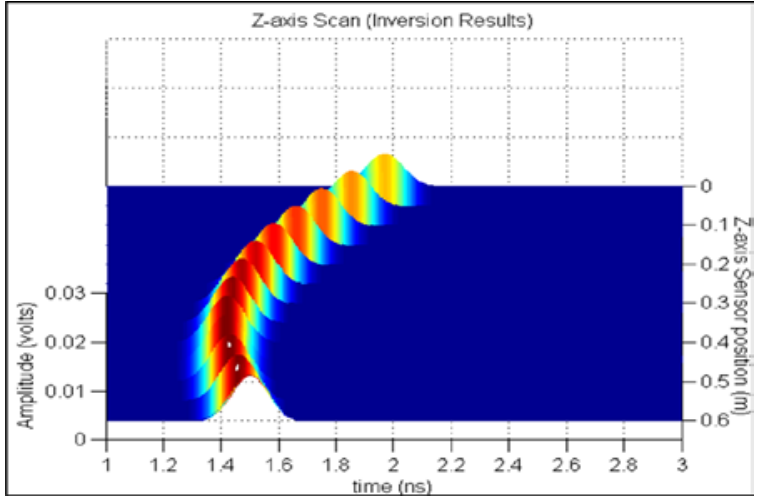

Fig 12(a) :. 3D representation of $Z$-axis SAR reconstructed response after range gate filtering for test posn. 1 .

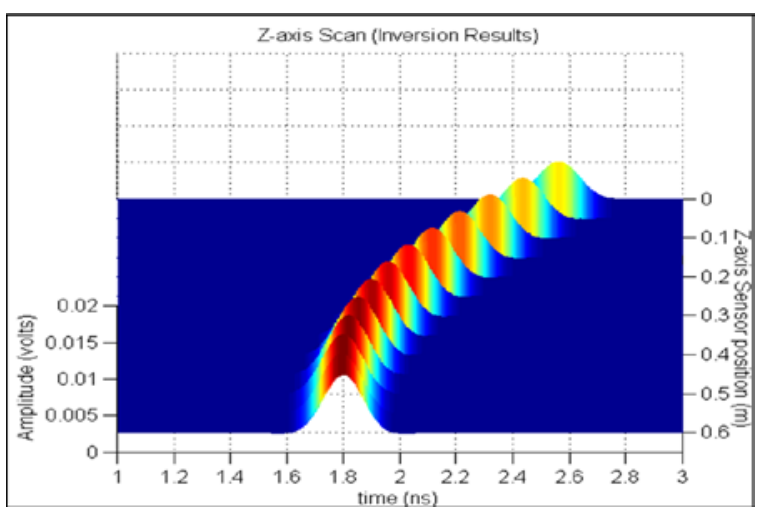

Fig 12(b) :. 3D representation of Z-axis SAR reconstructed response after range gate filtering for test posn. 2 .

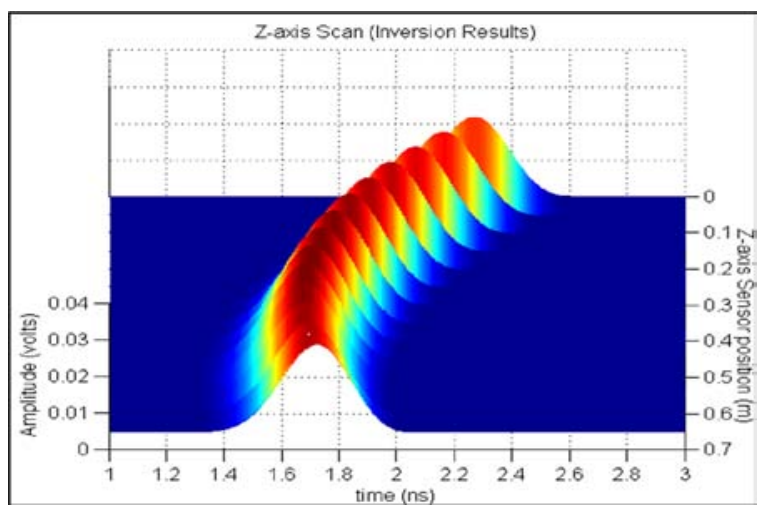

Fig 12(c) :. 3D representation of Z-axis SAR reconstructed response after range gate filtering for test posn. 3 .

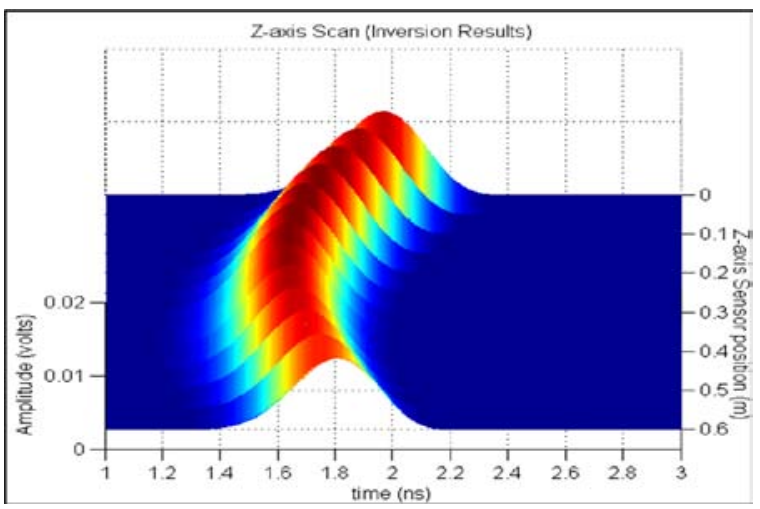

Fig 12(d) :. 3D representation of $\mathrm{Z}$-axis SAR reconstructed response after range gate filtering for test posn. 4 
A study of the 3-D figures reveals a close correlation between the reconstructed and measured pulse profiles, with typical dispersion induced pulse broadening being accurately replicated in the reconstructed pulses. Furthermore, the observed deviations in reconstructed pulse propagation delays, never amount to more than 100 ps of the absolute SAR measurement delay values, which vary between 1.5 to 2.5 ns. This close reproduction of the pulse characteristics, verifies the viability of the inversion process not only for sensor node position coordinates but also for the dielectric constant and antenna radiation pattern correction factor estimates. Any significant discrepancies in the data sets are largely confined to the relative amplitudes of the aforementioned measured and reconstructed pulse data, where deviations of up to $10 \%$ of the measured maxima are in evidence at some test positions.

\section{Inversion Error Analysis}

The fidelity of the pulse reconstruction algorithm may be evaluated independently for each measurement position via computation of the difference between measured and reconstructed pulses as a function of receive sensor position on the synthetic aperture array. This pulse difference 'error' as illustrated in Figs. 6(a) and 6(b) for $X$ and Z-axes respectively at test position 2 , is derived from the difference in amplitude between measured and reconstructed pulses of Figs. 5(a) and 5(b), which when plotted as a function of position, clearly shows a series of residual peaks following the iso-peak hyperbolic loci of the measured pulses. The numerical accuracy of the pulse reconstruction process may now be derived via normalisation of the RMS value of this difference with that of the measured pulse data over all $N$ time domain sampling points, i.e

$$
f_{e}=\sqrt{\frac{\sum_{n=1}^{N}\left|S_{\text {meas }}(n)-S_{\text {recon }}(n)\right|^{2}}{\sum_{n=1}^{N}\left|S_{\text {meas }}(n)\right|^{2}}}
$$

The error function of (19), upon evaluation at all SAR scan axes measurement positions for each of the emitting sensor test configurations, is plotted in Figs. $13 \& 14$ for $X$ and $Z$ axes respectively. A study of the resulting plots reveals fluctuations in the cumulative error function $f_{e}$ of between $6 \%$ and $22 \%$, with mean values not exceeding $20 \%$ for all $X$ and $Z$ 


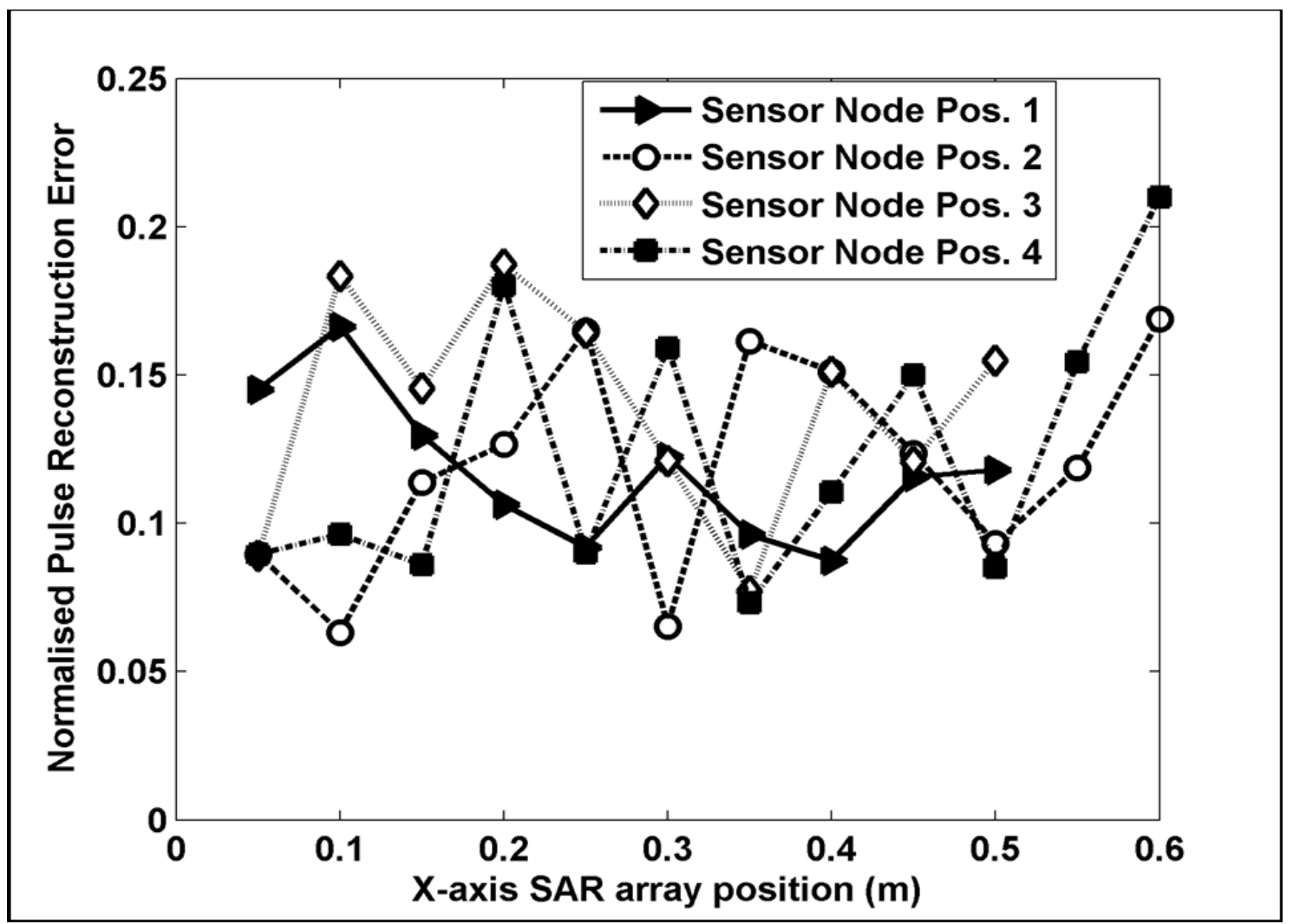

Fig 13 : Normalised RMS error between measured and reconstructed pulses at each X-axis scanning aperture measurement position.

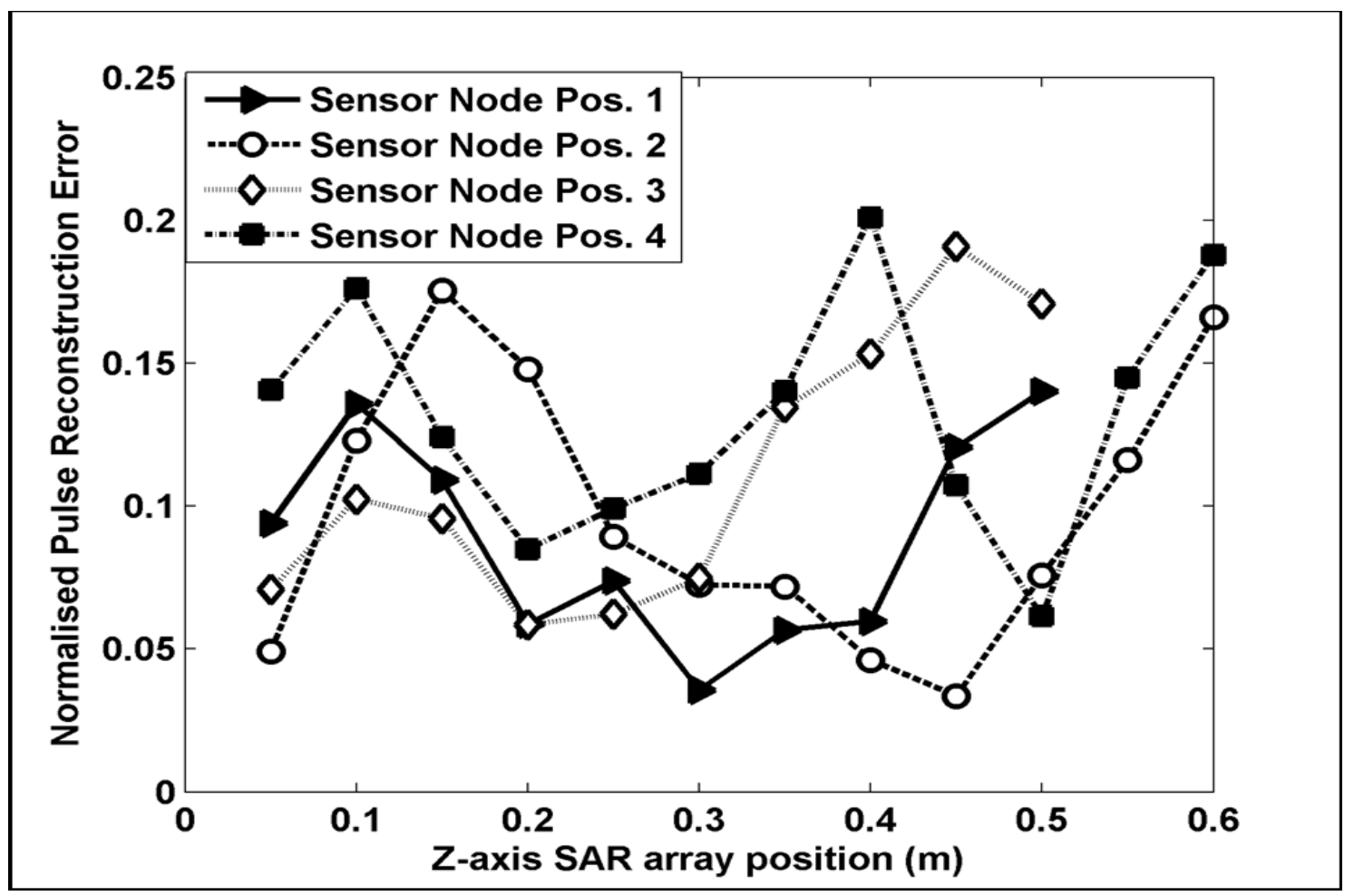

Fig 14 : Normalised RMS error between measured and reconstructed pulses at each Z-axis scanning aperture measurement position. 
axes scanning positions and emitter test configurations. It is evident from Figs. 5(a) \& (b), that the inversion process, in minimising the overall RMS error function of (17), has been forced to sacrifice the exact propagation delay alignment between measured and reconstructed pulses at certain positions in order to preserve it at others. The 1st SAR $X$ axis position is a particularly good example of this phenomenon, where a relatively modest temporal misalignment of approximately 100 ps has generated a significant statistical uncertainty approaching 20\%. This observed sensitivity of the SAR positional error function to temporal misalignment, attributable principally to the steep rate of change in pulse amplitude in the vicinity of the maxima, may well have masked the other potential error sources, namely i) polarisation and boresight squint offsets ii) vessel wall induced perturbations of the antenna pattern at the synthetic aperture edges.

\section{E. Inversion Algorithm - Positioning \& Permittivity Results}

Finally, the results of the inversion algorithm and complex permittivity estimates as conducted over the feasible range stipulated in Table 1, are summarised for all four sensor positions in Table 2. Here the first column provides the actual emitter position, defined in Fig. 1, as measured from the coordinate system origin, to the centre of its radiating ring monopole antenna, whilst columns 2 - 4 and 5 - 6 contain the inversion algorithm sensor coordinates and complex permittivity estimates respectively, and column 7 illustrates the inversion RMS error function from (17). A study of the data contained within, reveals a reasonable correlation between measured and reconstructed position coordinates in all cases, with only one of the coordinate positions exceeding the $6.2 \mathrm{~cm}$ and $5.8 \mathrm{~cm}$ down and cross range estimated resolution limits derived in Section 3E. Furthermore, a detailed analysis of the positioning data provided in Table 3, reveals an agreement between the calculated sensor coordinates and their manually measured counterparts of less than $7 \%$ for all but two of the position estimates. Note, this degradation in observed positioning accuracy, when compared with the air-spaced equivalent data in [3], which yielded positioning correlations within $4 \%$, is largely to be expected and emphasises the importance of an accurate determination of the host medium phase velocity [23, 24]. This modest loss of precision is principally due to the inhomogeneous and lossy nature of the wheat propagation media, where the associated spatial variation in permittivity coupled 
with dispersion induced pulse 'spreading' leads to an unavoidable loss of resolution with distance.

The statistical spread of the of complex permittivity estimates for all sensor test positions shown in Table $4,\left(\varepsilon^{\prime} \sim 2.26\right.$ to $2.68, \varepsilon^{\prime \prime}=0.15$ to 0.29$)$ is largely attributable to the inevitable spatial variations in the material properties of wheat over its $5.6 \mathrm{~m}^{3}$ measurement volume coupled with modest mechanical positioning errors. Furthermore, the well documented susceptibility of grain permittivity to packing density, pressure and moisture content [25] is a largely unstable, indeterminate property which cannot be accounted for in the inversion algorithm. Nonetheless, the mean and standard deviation estimates $\left(\varepsilon^{\prime} \sim 2.5\right.$ +/- 0.22, $\varepsilon "=0.204+/-0.056$ ) still yielded reasonable correlations with the typical values derived from the comprehensive studies attributable to Trabelsi and Nelson and published in [19, 20, 21]. For example [19], which provides an exhaustive study of the behaviour of wheat over a 0.1 to $10 \mathrm{GHz}$ frequency range for a $2.7 \%$ to $23.8 \%$ variation in moisture content using a combination of transmission line and resonant cavity measurement techniques, served as a useful guide as to the plausibility of the inversion algorithm derived permittivity data. A subsequent comparison in Table 4 of the estimated permittivity results with those extracted from the aforementioned study of [19] at 1.0 and $3.0 \mathrm{GHz}$ for UK laboratory based moisture levels of ( 10 \%) reveals only a marginal discrepancy between the range of measured permittivity values and the statistical variance of the inversion data exhibited in Table 4.

Note here, the credibility of these permittivity results was further reflected in the spread of antenna pattern ' $k$ ' correction values (1.7 to 2.1), where sensor test positions exhibiting the highest antenna gains, directivity values and ' $k$ ' factors, corresponded to those exhibiting the greatest dielectric constants and positioning resolutions, as predicted by the $\Delta x$ and $\Delta y$ resolution functions defined in (14) \& (15).

Table 2 : Summary of inversion algorithm positioning \& permittivity estimates

\begin{tabular}{|l|c|c|c|c|c|c|l|}
\hline Test Position (m)* & $\mathrm{X}(\mathrm{m})$ & $\mathrm{Y}(\mathrm{m})$ & $\mathrm{Z}(\mathrm{m})$ & $\varepsilon^{\prime}$ & $\varepsilon^{\prime \prime}$ & $k$ & $f e(\mathrm{Eqn} 17)$ \\
\hline 1: $(0.42,1.00,0.25)$ & 0.4093 & 1.072 & 0.266 & 2.685 & $235 \times 10^{-3}$ & 2.463 & $1.98 \times 10^{-5}$ \\
\hline 2: $(0.45,0.95,0.50)$ & 0.4431 & 0.927 & 0.545 & 2.408 & $293 \times 10^{-3}$ & 2.173 & $0.54 \times 10^{-5}$ \\
\hline 3: $(0.25,1.05,0.55)$ & 0.2710 & 1.015 & 0.509 & 2.265 & $202 \times 10^{-3}$ & 1.892 & $2.48 \times 10^{-5}$ \\
\hline $\mathbf{4 :}(0.58,0.80,0.40)$ & 0.5567 & 0.789 & 0.405 & 2.664 & $151 \times 10^{-3}$ & 2.443 & $1.37 \times 10^{-5}$ \\
\hline
\end{tabular}

*Manually measured values 
Table 3 : Analysis of inversion algorithm positioning data in Table 2

\begin{tabular}{|l|c|c|c|c|c|c|}
\hline \multirow{2}{*}{ Test Position (cm)* } & \multicolumn{3}{|c|}{ Inversion Position Error } & \multicolumn{3}{|c|}{ Normalised Position Error } \\
\cline { 2 - 7 } & $|\Delta \mathrm{X}|(\mathrm{cm})$ & $|\Delta \mathrm{Y}|(\mathrm{cm})$ & $|\Delta \mathrm{Z}|(\mathrm{cm})$ & $|\Delta \mathrm{X}| / \mathrm{X}$ & $|\Delta \mathrm{Y}| / \mathrm{Y}$ & $|\Delta \mathrm{Z}| / \mathrm{Z}$ \\
\hline 1: $(42.0,100,25.0)$ & 1.07 & 7.20 & 1.60 & $2.55 \%$ & $7.20 \%$ & $6.40 \%$ \\
\hline 2: $(45.0,95.0,50.0)$ & 0.69 & 2.30 & 4.50 & $1.53 \%$ & $2.42 \%$ & $9.00 \%$ \\
\hline 3: $(25.0,105,55.0)$ & 2.10 & 3.50 & 4.10 & $7.75 \%$ & $3.45 \%$ & $8.05 \%$ \\
\hline 4: (58.0, 80.0, 40.0) & 2.33 & 1.10 & 0.50 & $4.02 \%$ & $1.37 \%$ & $1.25 \%$ \\
\hline
\end{tabular}

*Manually measured values

Table 4 : Comparison of inversion algorithm permittivity estimates from Table 2 with published data attributable to [19].

\begin{tabular}{|c|c|c|c|c|c|c|c|}
\hline \multicolumn{2}{|c|}{$\begin{array}{c}\text { Permittivity Mean } \\
\text { (Positions 1-4) }\end{array}$} & \multicolumn{2}{|c|}{$\begin{array}{c}\text { Permittivity STD } \\
\text { Deviation (Posn. 1-4) }\end{array}$} & \multicolumn{2}{c|}{$\begin{array}{c}\text { Permittivity Data } \\
\text { from [19] @ 1 GHz }\end{array}$} & \multicolumn{2}{c|}{$\begin{array}{c}\text { Permittivity Data } \\
\text { from [19] @ GHz }\end{array}$} \\
\hline$\overline{\varepsilon^{\prime}}$ & $\overline{\varepsilon^{\prime \prime}}$ & $\sigma_{\varepsilon}{ }^{\prime}$ & $\sigma_{\varepsilon}{ }^{\prime \prime}$ & $\overline{\varepsilon^{\prime}}$ & $\overline{\varepsilon^{\prime \prime}}$ & $\overline{\varepsilon^{\prime}}$ & $\overline{\varepsilon^{\prime \prime}}$ \\
\hline 2.505 & 0.2039 & 0.2203 & 0.056 & 2.80 & 0.30 & 2.50 & 0.26 \\
\hline
\end{tabular}

\section{Conclusions}

This paper describes a novel inversion algorithm whose fundamental objective has been the determination of the position of wireless sensor nodes within the highly dispersive media encountered in wheat filled silos. Out of necessity, as the host medium phase velocity is initially unknown [24] it has also adapted the recently publicised equivalent free-space technique [4] to unquantified lossy dielectric environments, in order to simultaneously derive the node position coordinates, host medium permittivity and sensor antenna pattern correction factor. This permittivity extraction feature in particular, proved to be an essential extension of the original free space technique as this enabled the computation of both pulse propagation delay and attenuation in tandem with the sensor position.

As the original objective was to design a positioning array for a grain silo, where side access wasn't permitted, all positioning measurements were conducted from above. A synthetic aperture array was created via the positioning of single UWB ring monopole antennas at discrete intervals along a pair of orthogonal axes approximately $1 \mathrm{~m}$ in length. This method is partly analogous to GPR tomographic imaging applications and some of these design features have been incorporated into the uni-directional transmission configuration. A full tomographic SAR imaging exercise was not deemed necessary, as 
simultaneous identification of multiple sensors was not required, nor did we need to know the position of the walls, or other environmental features. This adapted inversion process, could best be described as 'imaging utilising line of sight propagation (LOS) only', where a 250 ps width Gaussian range gate has been applied to extract the LOS component with minimal sidelobe interference. The effective system bandwidth, estimated to be $~ 2.5 \mathrm{GHz}$, in conjunction with the $1 \mathrm{~m}$ synthetic aperture length, yielded respective down and cross range resolutions of better than $6.5 \mathrm{~cm}$ and $5.8 \mathrm{~cm}$ respectively over a $2 \mathrm{~m}$ diameter, $1.8 \mathrm{~m}$ depth cylindrical imaging volume.

The inversion process adopted a forward solution comprising a set of transfer functions, defining the propagation path between sensor emitter and each synthetic aperture receive position, in terms of position coordinates, complex permittivity and antenna correction factor (a parameter governing the distortion of the original radiation pattern by the material loss tangent). The transfer functions in determining both pulse delay and dispersion effects, offered an increase in complexity over the free space delay based counterpart in [4] and formed the basis of an optimisation process designed to minimise the misfit between measured and reconstructed pulses at all SAR array positions. The global solution for the above, in utilising all time domain sampling points, significantly enhanced the overall accuracy of the wheat based inversion process under extremely challenging propagation conditions, with the ensuing inversion localisation results correlating to within $+/-7 \mathrm{~cm}$ of the actual sensor positions.

For verification purposes, wheat permittivity values for typical UK moisture content variations were derived from data accessible in open literature [19, 20, 21]. On perusal, these were found to provide a reasonable agreement with the inversion solutions derived for a typical laboratory based moisture content of $~ 10 \%$ at selected frequencies of 1.0 \& 3.0 GHz within the positioning pulse bandwidth, where a correlation to within $10 \%$ of the published mean dielectric constant and loss tangent values was observed. It should be noted that the hygroscopic and inhomogeneous nature of the wheat propagation medium will engender significant variations in its permittivity values with environmental storage conditions, such as moisture content, packing compression and bulk density. Hence the permittivity values measured here although providing a reasonable agreement with the data attributable to Trabelsi and Nelson in [19, 20, 21], still only represents a macroscopic average over the dielectric constant over the measurement volume More detailed comparisons would however require knowledge of the environmental conditions and 
spatial variations of the wheat based material properties at the time of measurement - a level of detail deemed beyond the 'positioning oriented' scope of this paper at the time of writing.

- Potential future work could therefore comprise an extension of the inversion algorithm to incorporate an enhanced microwave tomographic imaging capability, comprising both spatial and frequency dependent variations in the bulk parameters of wheat. This could be coupled with further investigations into the susceptibility of the inversion process to environmental variations in the grain material properties, its effect on dispersive propagation conditions and positioning accuracy.

\section{Acknowledgements}

The authors would like to acknowledge the support of the EPSRC for funding the above research. Grant Reference: EP/D063523/1 : 'Wired \& Wireless Intelligent Networked Systems 2'.

\section{References}

[1] Hongting Jia, Takashi Takenaka, Toshiyuki Tanaka, 'Time-Domain Inverse Scattering Method for Cross-Borehole Radar Imaging'. IEEE Trans. on Geoscience and Remote Sensing, Volume: 36, Issue: 6, Nov 1998.

[2] P. Armstrong, 'Wireless Data Transmission of Networked Sensors in Grain Storage', 2003, ASAE Annual International Meeting, Sponsored by ASAE Riviera Hotel and Convention Center, Las Vegas, Nevada, USA, 27- 30 July 2003.

[3] J.G Davis, R. Sloan, A.J, Peyton, 'A Three-Dimensional Positioning Algorithm for Networked Wireless Sensors', IEEE Transactions on Instrumentation and Measurement, April 2011 Volume: 60, Issue: 4, pp. 1423 - 1432.

[4] J.G Davis, R. Sloan, A.J, Peyton, M. Bilal, 'A Positioning Algorithm for Wireless Sensors in Rich Multipath Environments', IEEE Microwave Wireless and Components letters, Volume 18, No. 9, pp. 644-646, Sept. 2008. 
[5] R Andrews, 'UWB Signal Sources, Antennas and Propagation,' Picosecond Pulse Labs, Boulder, CO, Application Note AN-14a, Aug. 2003.

[6] F.L. Teixeira, Weng Cho Chew, M. Straka, M.L. Oristaglio, T. Wang. 'FiniteDifference Time-Domain Simulation of Ground Penetrating Radar on Dispersive, Inhomogeneous, and Conductive Soils' IEEE Trans. on Geoscience and Remote Sensing, Volume. 36, No. 6, Nov 1998.

[7] W.C Chew, M. Straka, M.Oristaglio, T. Wang, 'Finite-difference time-domain simulation of ground penetrating radar on dispersive, inhomogeneous, and conductive soils', IEEE Trans. on Geoscience and Remote Sensing, Volume 36, Issue: 6, Nov 1998, pp. $1928-1937$.

[8] T.J. Cui, W. C. Chew, A. A. Aydiner, and S. Chen, 'Inverse scattering of twodimensional dielectric objects buried in a lossy earth using the distorted Born iterative method', IEEE Trans. Geoscience. Remote Sensing, Volume 39, pp. 339-346, Feb. 2001.

[9] G.A Meles, J.V der Kruk, S.A. Greenhalgh, J. R. Ernst, H. Maurer, and A. Green. 'A new vector waveform inversion algorithm for simultaneous updating of conductivity and permittivity parameters from combination crosshole/borehole-to-surface GPR data'. IEEE Trans. on Geoscience and Remote Sensing, Volume 48, Issue: 9, Sept 2010.

[10] J. Liang, C.C. Chiau, X. Chen, 'Design analysis of a circular ring monopole antenna'. Loughborough Antennas \& Propagation Conf. Proceedings, pp 128-131, 4th to 6th April 2005.

[11] J. Schroeder, S. Galler, and K. Kyamakya, 'A Low-Cost Experimental UltraWideband Positioning System', IEEE International Conference on Ultra-Wideband (IEEE Cat. No.05EX1170C), 2005, pp. 632-637.

[12] D.M. Pozar, Microwave Engineering, 2nd Ed., Wiley, 1998, pp. 672-674

[13] J.D Kraus, Antennas (2nd Edition), New York: McGraw-Hill, 1988, pp. 220-221. 
[14] H.J Griffiths, A. McAslan, 'Low frequency radar for buried target detection', Mineseeker Foundation Report, pp. 131-132, 2001.

[15] P. Millot, P. Berges, 'Ground based SAR imaging tool for the design of buried mine detectors', The Detection of Abandoned Land Mines: A Humanitarian Imperative Seeking a Technical Solution, EUREL International Conference on (Conf. Publ. No. 431), 1996, pp. 157- 159 .

[16] B. Panzner, A. Joestingmeier, A. Omar, S. Abbas. , 'Evaluation of SAR Focusing Methods for Ground Penetrating Radar',' (EUSAR), 2010 8th European Conference, 2010 , pp. 1 - 4 .

[17] K.C Ho, P.D. Gader, J.N Wilson; T. Glenn, 'On the use of energy density spectra for discriminating between landmines and clutter objects', IEEE Antennas and Propagation Society International Symposium, 2005 volume 3B, pp. 84 - 87.

[18] M. Nakhkash, Y. Huang, M.T.C Fang, 'Application of the Multi-Level Single Linkage Method to One-Dimensional EM Inverse Scattering', IEEE Transactions on Antennas and Propagation, 22p, July 2003.

[19] S.O. Nelson, S. Trabelsi, 'RF Sensing of Grain and Seed Moisture Content', IEEE Sensors Journal, Volume 1, no. 2, pp. 119.-126, August 2001.

[20] A.Kraszewski and S.O. Nelson, 'Wheat moisture content and bulk density determination by microwave parameters measurement', Can. Agricultural. Engineering, Volume 34, no. 4, pp. 327-335, 1992.

[21] S. Trabelsi and S.Nelson, 'Non-destructive sensing of physical properties of granular materials by microwave permittivity measurement', IEEE Transactions on Instrumentation and Measurement, Volume 55, No.8, June 2006, 1995, pp. 953-963.

[22] A. Ihamouten, G.Villain, X. Dérobert, 'Complex permittivity frequency variations from multi-offset GPR data of Hydraulic Concrete Characterization', IEEE Transactions on Instrumentation and Measurement, Volume 61, Issue: 6, 2012, pp. 1636 - 1648. 
[23] Chien-Ping Kao, Jing Li, Ying Wang, Huichun Xing, and Ce Richard Liu, 'Measurement of Layer Thickness and Permittivity Using a New Multilayer Model From GPR Data', IEEE Transactions on Transactions. on Geoscience and Remote Sensing, Volume 45, Issue: 8, August 2007.

[24] Z.Anxue, J.Yansheng, W.Wenbing, 'An algorithm for Estimating the Object Depth accurately with GPR ', Journal of Systems Engineering and Electronics, Volume 13, No. 3, September 2002, pp. 24 - 30.

[25] H.S. Chua, A.D. Haigh, F. Thompson and A.A.P. Gibson, ' Measurement of the wetmass, moisture content, volume and density of single wheat grain kernels', IEE Proceedings on Science Measurements \& Technology, Volume 151, No. 5, September 2004, pp. 384 - 388. 